\title{
A variational model for fracture and debonding of thin films under in-plane loadings
}

\author{
A. A. León Baldelli ${ }^{\mathrm{a}, \mathrm{b}, *}, \mathrm{~J}^{--F}$. Babadjian ${ }^{\mathrm{d}}$, B. Bourdine ${ }^{\mathrm{e}}$ D. Henao ${ }^{\mathrm{c}}$, C. Maurini ${ }^{\mathrm{a}, \mathrm{b}}$ \\ ${ }^{a}$ Institut Jean Le Rond d'Alembert (UMR-CNRS 1190), Université Paris 6 (UPMC), 4 place Jussieu, 75252 Paris, France \\ ${ }^{b}$ Institut Jean Le Rond d'Alembert (UMR-CNRS 7190), CNRS, \& place Jussieu, 75252 Paris, France \\ ${ }^{c}$ Facultad de Matemáticas, Pontificia Universidad Católica de Chile, Casilla 306, Correo 22, Santiago, Chile \\ ${ }^{d}$ Laboratoire Jacques Louis Lions (UMR-CNRS 7598), Université Paris 6 (UPMC), 4 place Jussieu, 75252 Paris, France \\ ${ }^{e}$ Department of Mathematics and Center for Computation $\&$ Technology, Louisiana State University, Baton Rouge LA \\ 70803, U.S.A
}

\begin{abstract}
We study fracture and delamination of a thin stiff film bonded on a rigid substrate through a thin compliant bonding layer. Starting from the three-dimensional system, upon a scaling hypothesis, we provide an asymptotic analysis of the three-dimensional variational fracture problem as the thickness goes to zero, using $\Gamma$-convergence. We deduce a two-dimensional limit model consisting of a brittle membrane on a brittle elastic foundation. The fracture sets are naturally discriminated between transverse cracks in the film (curves in 2D) and debonded surfaces (two-dimensional planar regions). We introduce the vectorial plane-elasticity case, applying the rigorous results established for scalar displacement fields, in order to numerically investigate the typical cracking scenarios encountered in applications. To this end, we formulate a reduced-dimension, rate-independent, irreversible evolution law for transverse fracture and debonding of thin film systems. Finally, we propose a numerical implementation based on a regularized formulation of the fracture problem via a gradient damage functional. We provide an illustration of the capabilities of the formulation exploring complex crack patterns in one and two dimensions, showing a qualitative comparison with geometrically involved real life examples.
\end{abstract}

Keywords: Thin films, fracture mechanics, asymptotic analysis, variational mechanics

\section{Introduction}

Cracking of thin films systems is often experienced in everyday life. Ceramic painted artefacts, coated materials, stickers, paintings and muds are some of the physical systems that exhibit the appearance of complex networks of cracks channeling through the topmost layer in the stack. In addition, the phenomenology is enriched by the possible interplay with mechanisms of spontaneous interfacial debonding. Although within the three-dimensional multilayer system cracks may appear anywhere and with arbitrary geometry, it is a common observation that cracks are either transverse, channeling through the film, or planar debonding surfaces at the interface. A comprehensive review of common fracture patterns may by found in (Hutchinson and Suo, 1992).

Within the framework of classical fracture mechanics, the propagation of crack tip(s) along a pre-defined crack path is obtained through a criterion of critical energy release rate. In their seminal paper, Hutchinson and Suo (1992) provide closed form computations of the energy release rate associated to isolated straight or kinked cracks for general layered materials. The concept of steady-state cracking is first formulated as the

\footnotetext{
* Corresponding author

Email address: leon@dalembert.upmc.fr (A. A. León Baldelli)
} 
condition for which the "crack driving force" is independent of the crack's size and it is attained as soon as the crack is long compared to the film thickness. Xia and Hutchinson (2000) propose a reduced two-dimensional model for a thin film system as an elastic membrane on an elastic foundation. Then, they investigate the steady-state propagation of isolated cracks and arrays of cracks, illustrate the interaction between parallel or perpendicular neighboring cracks and show, under additional hypotheses, the existence of a particular solution of a crack evolving along an Archimedean spiral. A comparison between the reduced model and the full three-dimensional non-homogeneous layer stack is carried out in Yin et al. (2008), validating the reduced model in the regime of stiff films over a compliant substrate. The presence of an elasto-plastic interface is investigated by McGuigan et al. (2003) and a family of visco-elasto-plastic effective laws for the bonding layer have been analyzed by Handge (2002).

From a numerical standpoint, fracture of thin films has been investigated via phenomenological springnetwork models by Crosby and Bradley (1997); Leung and Néda (2000); Sadhukhan et al. (2011), whilst Liang (2003) and Fan et al. (2011) proposed to tackle the problem by means of an extended finite leements discretisation. However, XFEM approaches still have difficulties in correctly describing crack branching, coalescence and nucleation. Neither of these works accounts for the interplay between channel cracking and debonding.

In the applied mathematics community, static fractures in single-layer thin films have been investigated by means of a $\Gamma$-convergence analysis that allows the identification of an effective reduced 2D model (Braides and Fonseca, 2001; Bouchitte et al., 2002). Babadjian (2006) studied the quasi-static evolution of cracks in thin films proving the convergence of the full three-dimensional evolution to the reduced two-dimensional one. These results are obtained considering a single-layer system resulting in cracks that are invariant in the thin direction. The dimension reduction of a bilayer thin film allowing for debonding at the interface has been investigated by Bhattacharya et al. (2002). The debonding is penalized by a phenomenological interfacial energy paying for the jump of the deformation at the interface. The limiting models are discussed according to the weight of interfacial energy. Rigorous derivations of decohesion-type energies have been given in (Ansini et al., 2007; Ansini, 2004) by means of a homogenization procedure. In these works the interfacial energy appears as the limit of a Neumann sieve, debonding being regarded as the effect of the interaction of two thin films through a suitably periodically distributed contact zone. More recently, Dal Maso and Iurlano (2013); Iurlano (2012); Focardi and Iurlano (2013) have also derived similar cohesive fracture models by means of an Ambrosio-Tortorelli approximation (Ambrosio and Tortorelli, 1992) involving an internal damage variable. Finally, several works have focused on the quasi-static evolution of debonding problems with a prescribed debonding zone. In particular, Roubíček et al. (2009) model the debonding phenomenon through an internal variable representing the volume fraction of adhesive contact between the layers.

In this paper we investigate the brittle thin film systems system within the framework of variational fracture mechanics (Francfort and Marigo, 1998; Bourdin et al., 2008), using techniques from the calculus of variations and the notion of variational convergence, which provide a key to reveal the consequences of the energy minimality requirement. We consider a thin film bonded to a rigid substrate through a compliant bonding layer. Under precise scaling hypotheses on the geometric and material properties of the film and the bonding layer, we use asymptotic analysis and $\Gamma$-convergence to deduce a limit two-dimensional model consisting of a membrane on an elastic foundation, similar to the one considered by Xia and Hutchinson (2000). The result includes the presence of cracks, showing that, in the limit, the energetically favored cracks are channeling cracks in the film and in-plane cracks in the bonding layer. We solve numerically the limit two-dimensional variational problem using a regularized formulation and a finite element discretization, which extends the one proposed by Bourdin et al. (2000). Then, we illustrate through several examples the complex crack patterns arising when applying inelastic strain in the film, showing the competing roles of traverse fracture and debonding. The results are obtained without any a priori hypotheses on the shape of the crack and without any ad-hoc criterion for crack initiation and propagation, the energy minimality requirements being the only guiding principle of the analytical and numerical work.

The present work may be regarded as a follow-up of León Baldelli et al. (2013), where the fracturing/debonding problem of a thin film has been studied analytically in one dimension. Recently, Mesgarnejad et al. (2013) considered the fracture of thin films in bending, but without delamination, reporting numerical 
results obtained with the same methods used here. Corson et al. (2010) present an interesting phase-field approach to study hierarchical patterns under mechanical stresses with a model that for many aspects is similar to the one proposed in the present paper. In all these works, the brittle thin-film model is postulated without deducing it as a limit of a three-dimensional brittle system.

The paper is organized as follows. In Section 2 we introduce the three-dimensional system, from which we derive an asymptotic two-dimensional reduced model in brittle elasticity. We define the three-dimensional total energy in the framework of linear elasticity with free discontinuities and state the variational principle that rules the static and evolution problems. In Section 3 we present the main result of dimensional reduction in the case of scalar elasticity, namely Theorem 3.1, along with its fundamental implications from a mechanical standpoint. The proof of the mathematical result is reported in Appendix B, to favor readability. In Section 4, we apply to vectorial elasticity the model rigorously derived in the scalar case (without proof) and formulate the corresponding quasi-static evolution problem. Section 5 presents the regularized formulation and the numerical implementation. The results of several numerical experiments are reported in Section 6. Conclusions are drawn in Section 7.

\section{Formulation of the problem}

\subsection{Preliminaries and notation}

We use Einstein's summation convention throughout the paper, unless specified otherwise. Roman and greek subscripts denote components of tensors of rank 1,2 or 3 , respectively spanning the sets $\{1,2,3\}$ and $\{1,2\}$. We deal with "thin" domains in $n \mathrm{D}(n=2,3)$, i.e. with domains for which one characteristic dimension is much smaller than the remaining $n-1$. We denote by $\Omega$ the reference configuration of a threedimensional brittle elastic (possibly non-homogeneous) cylinder whose basis is $\omega \subset \mathbb{R}^{2}$. The associated energy density function is denoted by $W: \mathbb{R}^{m \times n} \rightarrow \mathbb{R}$, where $\mathbb{R}^{m \times n}$ stands for the set of real $m \times n$ tensors. In the sequel we deal with $3 \mathrm{D}$ elasticity $(m=n=3), 2 \mathrm{D}$ plane elasticity $(m=n=2)$ and scalar elasticity $(m=3, n=1)$. Accordingly, the linearized gradient of the deformation is $\epsilon(u):=\frac{1}{2}\left(\nabla u+\nabla^{\top} u\right)=$ $\frac{1}{2}\left(\partial_{j} u_{i}+\partial_{i} u_{j}\right)$ in $3 \mathrm{D}$ elasticity, $\epsilon(u):=\frac{1}{2}\left(\nabla^{\prime} u+\nabla^{\prime \top} u\right):=\frac{1}{2}\left(\partial_{\alpha} u_{\beta}+\partial_{\beta} u_{\alpha}\right)$ in $2 \mathrm{D}$ elasticity - the prime sign indicating derivatives with respect to the in-plane coordinates - and reduces to the gradient $\epsilon(u)=\nabla u$ in scalar elasticity. We denote by a dot the scalar (inner) product. We shall use the usual notations for function spaces: $H^{1}\left(\Omega ; \mathbb{R}^{n}\right), L^{2}\left(\Omega ; \mathbb{R}^{n}\right), L^{\infty}\left(\Omega ; \mathbb{R}^{n}\right)$ and $S B V\left(\Omega ; \mathbb{R}^{n}\right)$ are respectively the space of square integrable functions with squared integrable derivatives, the Lebesgue space of square integrable functions, the space of functions with finite sup-norm and the space of special functions of bounded variations, defined on the set $\Omega$ and with values in $\mathbb{R}^{n}$. Whenever $n=1$, for simplicity, we will use the abbreviated notations $H^{1}(\Omega), L^{2}(\Omega), L^{\infty}(\Omega)$ and $S B V(\Omega)$. We mark with a superposed tilde dimensional functions, domains and operators. We shall distinguish between the strong and the weak problem of brittle films. The strong problem will always be defined for admissible displacements in a suitable subspace of the usual Sobolev space $H^{1}(\cdot)$. For the weak problem, we denote by a calligraphic capital letter, e.g. $\mathcal{C}(\cdot)$, the subset of special functions of bounded variations $S B V(\cdot)$ of admissible displacements. In favor of legibility, we commit an abuse of notation allowing us to label different functions with the same symbol, provided that they have a different number of arguments, so that e.g. no ambiguity shall arise between the two different functions $P(u, \Gamma)$ and $P(u)$.

\subsection{The three-dimensional brittle system}

The three-dimensional model system is sketched in Figure 1. A thin film $\tilde{\Omega}_{f}=\omega \times\left(0, h_{f}\right)$ is bonded to a rigid substrate $\tilde{\Omega}_{s}=\omega \times\left(-h_{s},-h_{b}\right)$ by means of a bonding layer $\tilde{\Omega}_{b}=\omega \times\left[-h_{b}, 0\right]$, where $\omega \subset \mathbb{R}^{2}$ is a bounded open set with Lipschitz boundary. In all that follows, the subscript $b$ indicates quantities relative to the bonding layer and $f$ to the film. The interface between the latter and the substrate is denoted by $\tilde{\Sigma}=\omega \times\left\{-h_{b}\right\}$. We assume the two layers to be isotropic and linearly elastic, the elasticity tensor being characterized by two material constants, e.g. the Lamé parameters $\left(\lambda_{f}, \mu_{f}\right)$ and $\left(\lambda_{b}, \mu_{b}\right)$ respectively for the coating film and bonding layer. We denote by $\tilde{\Omega}:=\tilde{\Omega}_{f} \cup \tilde{\Omega}_{b} \cup \tilde{\Omega}_{s}$ the full medium composed of the film, the bonding layer and the substrate. 


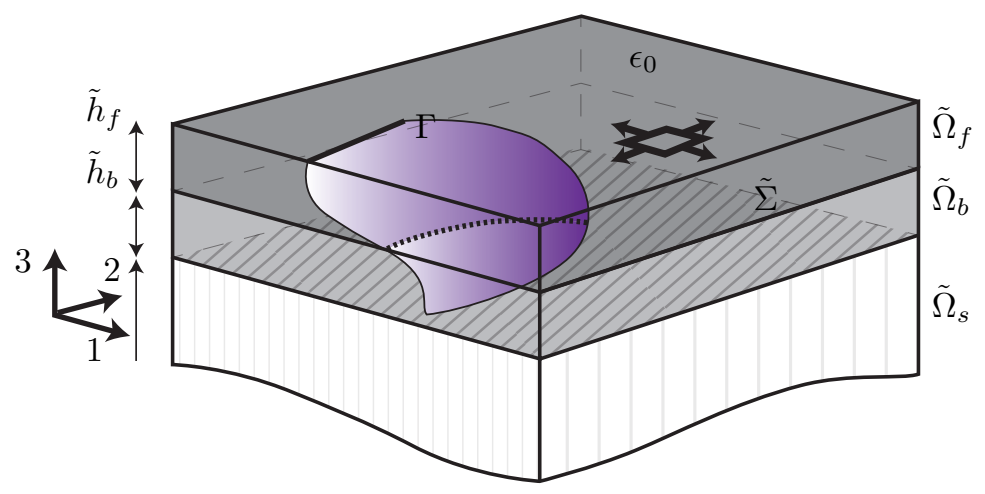

Figure 1: The three-dimensional model of the brittle system: a thin film $\Omega_{f}$ is bonded to a rigid substrate $\Omega_{s}$ via a bonding layer $\Omega_{b}$. Crack surfaces are noted $\Gamma$.

We consider two types of loading modes. The first is the displacement imposed at the interface $\tilde{\Sigma}$ by the substrate (Dirichlet boundary condition). We denote it by $w: \tilde{\Sigma} \rightarrow \mathbb{R}^{3}$. Considering the substrate infinitely stiff with respect to the film and bonding layer, the displacement $w(\tilde{x})$ at the interface can be identified as the displacement that the structure would undergo neglecting the presence of the surface coating layers under structural loads. The second load type is an inelastic strain $\epsilon_{0}: \tilde{\Omega}_{f} \rightarrow \mathbb{R}^{3 \times 3}$. Physically, it may rise due to thermal loadings, humidity or drying processes, just to note some of the possible multi-physical couplings that may take place. The inelastic strain $\epsilon_{0}(\tilde{x})$ is interpreted as the strain that the film and the bonding layer would undergo if they were free from compatibility constraints. We study the specific case of in-plane loads, i.e. loads for which only the in-plane components $\left(\epsilon_{0}\right)_{\alpha \beta}$ and $(w)_{\alpha}$ are non-vanishing, and when the inelastic strains are constant through the thickness of each layer. We choose not to account for all the multi-physical phenomena that may induce shrinking and model both loads as independent given parameters.

We represent cracks by discontinuity surfaces of the displacement field, we denote them by $\Gamma$ and let them free to appear anywhere inside the body without any a priori geometric restriction. Hence, cracks may be any set $\Gamma \subset \tilde{\Omega}$ of finite 2-dimensional Hausdorff measure. For clarity, the ones inside the film are denoted by $\Gamma_{f}:=\Gamma \cap \tilde{\Omega}_{f}$ whereas those inside the bonding layer are $\Gamma_{b}:=\Gamma \cap \tilde{\Omega}_{b}$. We assume that the cracks are created at the expense of a surface energy of Griffith-type, i.e. proportional to the measure of the crack surface by a material constant, the toughness $G_{c}$. We define it as follows:

$$
\mathcal{S}(\Gamma):=\int_{\Gamma} G_{c}(x) d \mathcal{H}^{2}, \quad G_{c}(x):= \begin{cases}G_{f}, & \text { if } x \in \tilde{\Omega}_{f}, \\ G_{b}, & \text { if } x \in \tilde{\Omega}_{b},\end{cases}
$$

where $\mathcal{H}^{2}$ is the Hausdorff surface measure.

In the framework of three-dimensional linear elasticity, the space of admissible displacements is that of square integrable vector fields with square integrable derivatives, defined on the sound part of the body $\tilde{\Omega} \backslash \Gamma$ and satisfying the boundary condition on the extended domain $\tilde{\Omega}_{s}$, namely:

$$
H_{w}^{1}\left(\tilde{\Omega} \backslash \Gamma ; \mathbb{R}^{3}\right):=\left\{v \in H^{1}\left(\tilde{\Omega} \backslash \Gamma ; \mathbb{R}^{3}\right), v=w \text { on } \tilde{\Omega}_{s}\right\}
$$

To each admissible field we associate the three-dimensional potential elastic energy:

$$
\mathcal{P}(u, \Gamma):=\frac{1}{2} \int_{\tilde{\Omega} \backslash \Gamma} W\left(\epsilon(u(x))-\epsilon_{0}(x)\right) \mathrm{d} x
$$

where the elastic energy density $W(\epsilon)$ reads:

$$
W(\epsilon)=\lambda(x) \operatorname{tr}(\epsilon)^{2}+2 \mu(x) \epsilon \cdot \epsilon .
$$


In the last expression $\lambda(x)$ and $\mu(x)$ are the piecewise constant elasticity parameters of the non-homogeneous body, namely:

$$
(\lambda, \mu)(x):= \begin{cases}\left(\lambda_{f}, \mu_{f}\right) & \text { if } x \in \tilde{\Omega}_{f}, \\ \left(\lambda_{b}, \mu_{b}\right) & \text { if } x \in \tilde{\Omega}_{b} .\end{cases}
$$

Finally, the total energy of the thin film system is the sum of the elastic and the surface energies, namely:

$$
\mathcal{E}(u, \Gamma):=\mathcal{P}(u, \Gamma)+\mathcal{S}(\Gamma) .
$$

\subsection{Static fracture problem}

Following the variational approach to fracture (Francfort and Marigo, 1998; Bourdin et al., 2008), we introduce the following definition of static fracture problem. The label static emphasizes that the solution is sought for a fixed load intensity and crack irreversibility does not play any role.

Problem 1 (Static problem for the three-dimensional brittle system). The static three-dimensional problem of brittle thin film systems consists in finding, for a given load intensity $\left(\epsilon_{0}, w\right)$, crack sets $\Gamma$ and (possibly) discontinuous displacement fields $u \in H_{w}^{1}\left(\tilde{\Omega} \backslash \Gamma ; \mathbb{R}^{3}\right)$ that solve the following minimization problem

$$
\inf \left\{\mathcal{E}(u, \Gamma): \quad \Gamma \subset \tilde{\Omega}, u \in H_{w}^{1}\left(\tilde{\Omega} \backslash \Gamma ; \mathbb{R}^{3}\right)\right\}
$$

i.e. that satisfy the following global minimality condition:

$$
\mathcal{E}(u, \Gamma) \leq \mathcal{E}(\hat{u}, \hat{\Gamma}), \quad \forall \hat{\Gamma} \subset \tilde{\Omega}, \forall \hat{u} \in H_{w}^{1}\left(\tilde{\Omega} \backslash \hat{\Gamma} ; \mathbb{R}^{3}\right) .
$$

The relevant minimization framework when dealing with Griffith-type surface energies is that of global minimization. With such surface energies, the elastic state is a local minimizer regardless of the loading magnitude (see Chambolle et al., 2007, 2010), so that cracks can never nucleate in a body without strong singularities. In some sense, a pre-existing crack or a geometric singularity on the boundary is required in order to release enough elastic energy to balance increase of surface energy. This issue may be mitigated by the introduction of more refined models, such as cohesive or gradient damage models (see Bourdin et al., 2008; Pham et al., 2011b, and also Section 5.3.).

\subsection{Quasi-static evolution problem}

Unlike in the static case, irreversibility plays a fundamental role in evolution problems. Upon prescribing a load history, parametrized by a scalar $t$, the three-dimensional evolution problem for brittle thin film systems consists in finding displacements and crack sets verifying a variational statement under the irreversibility constraint which forbids self-healing of cracks during the loading process. In the framework of variational fracture mechanics, the energetic formulation of evolution problem falls into the class of rateindependent processes as studied in Mielke (2005). The rate-independence implies that solutions to the evolution problem are stable under a reparametrization of the loading parameter, i.e. solutions are the same regardless of the velocity of the load. In this context we allow ourselves to interpret the arbitrarily increasing loading parameter $t$ as a "time" variable. We focus here in the time-discrete formulation of the problem. The reader can refer to Mielke (2005) for a time-continuous formulation.

Problem 2 (Time-discrete evolution problem for the three-dimensional system). Let $0=t_{0} \leq t_{1} \leq \ldots \leq$ $t_{N}=T$ be the discretization of the time interval $[0, T]$ into $N$ time steps. A time-discrete quasi-static evolution of the displacement field and crack set of the three-dimensional system is a mapping $t_{i} \mapsto\left(u^{i}, \Gamma^{i}\right)$ that, given the initial crack state $\Gamma^{0}$ and the loading history $\left(\epsilon_{0}^{i}, w^{i}\right)$, verifies the following global unilateral minimality conditions $\forall i \in 1, \ldots, N$ :

$$
\begin{aligned}
\Gamma^{i} \supseteq \Gamma^{i-1}, \\
\mathcal{E}\left(u^{i}, \Gamma^{i}\right) \leq \mathcal{E}(u, \Gamma) \quad \forall \Gamma \text { with } \Gamma^{i-1} \subseteq \Gamma \subset \tilde{\Omega}, \quad \forall u \in H_{w^{i}}^{1}\left(\tilde{\Omega} \backslash \Gamma ; \mathbb{R}^{3}\right), \text { with } \epsilon_{0}=\epsilon_{0}^{i} .
\end{aligned}
$$

These conditions are equivalent to require $\left(u^{i}, \Gamma^{i}\right)$ to be a solution of the minimization problem

$$
\inf \left\{\mathcal{E}(u, \Gamma): \quad \Gamma^{i-1} \subseteq \Gamma \subset \tilde{\Omega}, u \in H_{w^{i}}^{1}\left(\tilde{\Omega} \backslash \Gamma ; \mathbb{R}^{3}\right)\right\}
$$


In the problem above, the condition (7a) ensures the irreversibility of the fracturing process and prevent crack self-healing, whilst condition (7b) requires the constrained energy minimality of the solution at a given time step among all the admissible competitors. The weak regularity assumption on the crack set (finiteness of the two-dimensional Hausdorff measure) will allow the crack to take complex spatial shapes, branch, intersect and coalesce.

\subsection{Scaling hypotheses}

A natural "small parameter", denoted henceforth by $\varepsilon$, appears in thin film systems as the ratio between the thickness of the surface coating and its in-plane dimension, say $L$. In addition, thin film systems often exhibit abrupt variations of the material parameters characterizing the material behavior of the different layers, spanning several orders of magnitude. The separation of length scales and the material inhomogeneity renders the numerical solution computationally costly and motivates the derivation of reduced two-dimensional limit models. In order to study the asymptotic behavior of the brittle system we consider a specific scaling law for the elastic and geometric quantities and for the material toughnesses, as functions of the small parameter $\varepsilon=h_{f} / L$. Amongst all possible choices, we focus on the case where the layers' thicknesses $h_{b}$ and $h_{f}$ are of the same order of magnitude and the bonding layer is more compliant and weaker than the coating film. We formalize the two hypotheses by the following scaling laws on the geometric and elastic constants:

Hypothesis 1 (Scaling law of thicknesses and elastic moduli). Being

$$
\varepsilon:=\frac{h_{f}}{L} \ll 1
$$

we assume that the thickness of the bonding layer $h_{b}$ scales with $\varepsilon$ as the thickness of the film $h_{f}$, and that the ratio between the elastic constant of the bonding layer and that of the film scales as $\varepsilon^{2}$ :

$$
\frac{h_{b}}{h_{f}}=\rho_{h}, \quad \frac{\mu_{b}}{\mu_{f}}=\varepsilon^{2} \rho_{\mu}, \quad \frac{\lambda_{b}}{\lambda_{f}}=\varepsilon^{2} \rho_{\lambda}
$$

where $\rho_{h}, \rho_{\lambda}, \rho_{\mu}$ are dimensionless coefficients independent of $\varepsilon$.

Remark 2.1. Hypothesis 1 can be translated into the assumption that the shear energy of the bonding layer and the membrane energy of the film are of the same order of magnitude. A more general scaling law than (9) reflecting this coupling can be envisaged. However, one can show that the asymptotic elastic limit of the whole class of systems is the same. In this sense, a system satisfying (9) is taken as a representative of the whole class.

Regarding the material toughness, we focus on the interesting case where an interplay between cracking within the film and in the bonding layer takes place, the energy of transverse cracks in the film and the energy of in-plane cracks in the bonding layer being of the same order of magnitude. The former is of order $G_{f} h_{f} L$ and the latter of order $G_{b} L^{2}$, this hypothesis translates in the following scaling of the material toughnesses:

Hypothesis 2 (Scaling law of toughnesses). Being $\varepsilon=h_{f} / L$, the fracture toughnesses of the film $G_{f}$ and of the bonding layer $G_{b}$ are such that

$$
\frac{G_{b}}{G_{f}}=\varepsilon \rho_{G}
$$

where $\rho_{G}$ is a dimensionless constant independent of $\varepsilon$. 


\section{Reduced Model in Scalar Elasticity}

Our goal now is to study the asymptotic behavior $\varepsilon \searrow 0$ of a system verifying the geometric and constitutive Hypotheses 1 and 2. We expect a non-trivial coupling between the elastic energies of the layers and the competition between film and bonding layer cracks in order to release the stored elastic energy.

In this section we show a rigorous approximation result in the framework of scalar elasticity, i.e. when the displacement field is a scalar function. Although the case of scalar elasticity has an obvious physical meaning only in the case of two-dimensional domains (anti-plane elasticity), we consider the 3D case. This allows us to get, without any further mathematical burden, a clearer analogy to the full vector-valued problem. We prove that under the scaling hypotheses of Section 2.5, the three-dimensional brittle fracture problem admits a limit two-dimensional representation, providing the geometric characterization of crack surfaces both in the film and in the bonding layer, the shape of the optimal displacement field through the thickness and the two-dimensional limit energy. The result is based on the direct method of the calculus of variations and on $\Gamma$-convergence techniques. To make the mathematical argument rigorous, the problem is put into a convenient variational setting by considering displacement fields in the functional space $S B V$ of special functions of bounded variation (see Appendix A), as is classical in free-discontinuity problems Ambrosio et al. (2000). A displacement field $u$ in that space may be discontinuous and have jumps on a set denoted by $J_{u}$ which can be identified with the cracks. Outside the jump set, such displacements have an (approximate) gradient denoted by $\nabla u$, which is essentially the regular part of the differential of $u$. The reader is referred to Ambrosio et al. (2000) for a precise definition of this space and associated problems.

We formulate the problem on an extended domain $\tilde{\Omega}=\tilde{\Omega}_{f} \cup \tilde{\Omega}_{b} \cup \tilde{\Omega}_{s}$ including a portion of the substrate, say $\tilde{\Omega}_{s}:=\tilde{\omega} \times\left(-2 h_{b},-h_{b}\right)$. The space of all admissible displacements is given by the space

$$
\mathcal{C}_{w}(\tilde{\Omega}):=\left\{\tilde{u} \in S B V(\tilde{\Omega}): \tilde{u}=w \text { a.e. in } \tilde{\Omega}_{s}, \text { and }\|\tilde{u}\|_{L^{\infty}} \leq M\right\} .
$$

Note that the Dirichlet datum $w$ is a priori only defined at the interface $\tilde{\Sigma}$ between the substrate and the bonding layer. We implicitly extend it constantly to the whole domain $\tilde{\Omega}$ so that, from now on, $w$ is identified to a function on $\tilde{\Omega}$ independent of the out of plane variable. Therefore, the boundary condition $\tilde{u}=w$ on $\tilde{\Sigma}=\omega \times\left\{-h_{b}\right\}$ is expressed on the whole set of finite volume $\tilde{\Omega}_{s}$. We further assume that every deformation takes place in a container $K$ which is a compact subset of $\mathbb{R}^{3}$, i.e. $\|\tilde{u}\|_{L^{\infty}(\Omega)} \leq M$ for some fixed constant $M>0$, and $\|w\|_{L^{\infty}\left(\Omega_{s}\right)} \leq M$. The latter hypothesis can be removed at the expense of some additional technicalities (see Dal Maso et al. (2005)).

\subsection{Variational formulation in $S B V$ and rescaling of the energy}

In the case of scalar elasticity, the elastic energy density (3) reduces to $\mu_{\varepsilon}|\nabla u|^{2}$. To state the variational problem in a framework well suited for the mathematical analysis we rewrite the energy functional, defined for admissible displacements in $\tilde{u} \in \mathcal{C}_{w}(\tilde{\Omega})$, in the following form:

$$
\tilde{\mathcal{E}}_{\varepsilon}(\tilde{u})=\frac{\mu_{f}}{2} \int_{\tilde{\Omega}_{f}}\left|\nabla \tilde{u}-\epsilon_{0}\right|^{2} \mathrm{~d} \tilde{x}+\frac{\mu_{b}}{2} \int_{\tilde{\Omega}_{b}}\left|\nabla \tilde{u}-\epsilon_{0}\right|^{2} \mathrm{~d} \tilde{x}+G_{f} \mathcal{H}^{2}\left(J_{\tilde{u}} \cap \tilde{\Omega}_{f}\right)+G_{b} \mathcal{H}^{2}\left(J_{\tilde{u}} \cap \tilde{\Omega}_{b}\right) .
$$

As is customary in asymptotic methods (Lions, 1973; Ciarlet, 1997), we apply a change of variables in order to formulate the problem on a domain independent of the small parameter $\varepsilon$. The new non-dimensional space variable $x$ is defined by the following anisotropic scaling:

$$
x=\left(x^{\prime}, x_{3}\right)=\left(\tilde{x}^{\prime}, \frac{\tilde{x}_{3}}{\varepsilon}\right), \quad \text { with } \quad \tilde{x}^{\prime}=\left(\tilde{x}_{1}, \tilde{x}_{2}\right), \quad x^{\prime}=\left(x_{1}, x_{2}\right) .
$$

In the new variables, the film, the bonding layer and the substrate occupy the domains $\Omega_{f}=\omega \times(0, L)$, $\Omega_{b}=\omega \times\left[-\rho_{h} L, 0\right]$ and $\Omega_{s}:=\omega \times\left(-2 \rho_{h} L,-\rho_{h} L\right)$, respectively. In that configuration, the new displacement $u$ is defined by:

$$
u(x)=\tilde{u}(\tilde{x})
$$


and the gradient operator may be decomposed into its dimensionless in-plane and out-of-plane component as follows:

$$
\nabla \tilde{u}(\tilde{x})=\left(\nabla^{\prime} u(x), \frac{1}{\varepsilon} \partial_{3} u(x)\right), \quad \text { where } \quad \nabla^{\prime}=\left(\frac{\partial}{\partial x_{1}}, \frac{\partial}{\partial x_{2}}\right), \quad \partial_{3}=\frac{\partial}{\partial x_{3}} .
$$

Moreover, denoting respectively by $\nu_{\tilde{u}}$ and $\nu_{u}$ the unit normal to the jump sets $J_{\tilde{u}}$ and $J_{u}$ before and after the change of variables, the surface measure of $J_{\tilde{u}}$ is written as:

$$
\mathcal{H}^{2}\left(J_{\tilde{u}}\right)=\int_{J_{\tilde{u}}}\left|\nu_{\tilde{u}}\right| d \mathcal{H}^{2}=\int_{J_{u}}\left|\left(\varepsilon \nu_{u}^{\prime}, \nu_{u 3}\right)\right| d \mathcal{H}^{2}, \quad \text { where } \nu_{u}^{\prime}=\left(\nu_{u 1}, \nu_{u 2}\right) .
$$

Hence, the total energy (up to a multiplicative constant $1 / \varepsilon$ ) reads as

$$
\begin{aligned}
\mathcal{E}_{\varepsilon}(u):=\frac{\tilde{\mathcal{E}}_{\varepsilon}(\tilde{u})}{\varepsilon}= & \frac{\mu_{f}}{2}\left(\int_{\Omega_{f}}\left(\left|\nabla^{\prime} u-\epsilon_{0}^{\prime}\right|^{2}+\frac{1}{\varepsilon^{2}}\left(\partial_{3} u\right)^{2}\right) \mathrm{d} x+\rho_{\mu} \int_{\Omega_{b}}\left(\varepsilon^{2}\left|\nabla^{\prime} u-\epsilon_{0}^{\prime}\right|^{2}+\left(\partial_{3} u\right)^{2}\right) \mathrm{d} x\right) \\
& +G_{f}\left(\int_{J_{u} \cap \Omega_{f}}\left|\left(\nu_{u}^{\prime}, \frac{\nu_{u 3}}{\varepsilon}\right)\right| \mathrm{d} \mathcal{H}^{2}+\rho_{G} \int_{J_{u} \cap \Omega_{b}}\left|\left(\varepsilon \nu_{u}^{\prime}, \nu_{u 3}\right)\right| \mathrm{d} \mathcal{H}^{2}\right) .
\end{aligned}
$$

In the previous expression of the energy we have supposed for simplicity that the inelastic strain is of the form $\epsilon_{0}=\left(\epsilon_{0}^{\prime}, 0\right)$, where $\epsilon_{0}^{\prime} \in L^{2}\left(\Omega ; \mathbb{R}^{2}\right)$. Note that this change of variable does not affect the imposed boundary displacement $w$ since it is independent of the out of plane variable. Identifying $w$ with a function defined only on the plane, we henceforth assume that $w \in H^{1}(\omega) \cap L^{\infty}(\omega)$. Consequently, the rescaled space of all admissible displacements is:

$$
\mathcal{C}_{w}(\Omega):=\left\{u \in S B V(\Omega): u=w \text { a.e. in } \Omega_{s}, \text { and }\|u\|_{L^{\infty}\left(\Omega_{f}\right)} \leq M\right\}
$$

is independent of $\varepsilon$. The static fracture mechanics problem is reformulated as follows.

Problem 3 (Static problem for scalar elasticity. Weak formulation). For a given load intensity $\left(\epsilon_{0}, w\right)$, find $u \in \mathcal{C}_{w}(\Omega)$ that satisfy the following global minimality condition:

$$
\mathcal{E}_{\varepsilon}(u) \leq \mathcal{E}_{\varepsilon}(\hat{u}), \quad \forall \hat{u} \in \mathcal{C}_{w}(\Omega)
$$

Standard arguments ensure that this problem is well posed for fixed $\varepsilon$, in the sense that there exists at least a solution. The result is formalized by the following proposition. For the proof, the reader can refer to Ambrosio et al. (2000).

Proposition 3.1. (Existence of minimizers at fixed $\varepsilon)$ For each $\varepsilon>0, \epsilon_{0}^{\prime} \in L^{2}\left(\omega ; \mathbb{R}^{2}\right)$ and $w \in H^{1}(\omega) \cap$ $L^{\infty}(\omega)$, there exists a minimizer

$$
u_{\varepsilon} \in \underset{u \in \mathcal{C}_{w}(\Omega)}{\arg \min } \mathcal{E}_{\varepsilon}(u)
$$

\subsection{Limit model for $\varepsilon \rightarrow 0$}

Our aim is to determine a limit functional $\mathcal{E}_{0}$ and an associated minimization problem formulated on the two-dimensional domain $\omega$ that approximates the full three-dimensional problem for small $\varepsilon$. This energy will turn out to be finite over a set of kinematically admissible displacements which are invariant in the film with respect to the out-of-plane direction. They will be identified with displacements defined only on the plane $\omega$ and spanning the set:

$$
\mathcal{C}(\omega)=\left\{u \in S B V(\omega):\|u\|_{L^{\infty}(\omega)} \leq M\right\}
$$

Note that the approximate gradient of such displacements is given by $\nabla u=\left(\nabla^{\prime} u, 0\right)$, while the jump set is of the form $J_{u}=J_{u}^{\prime} \times(0, L)$.

The main result is summarized in the following theorem: 


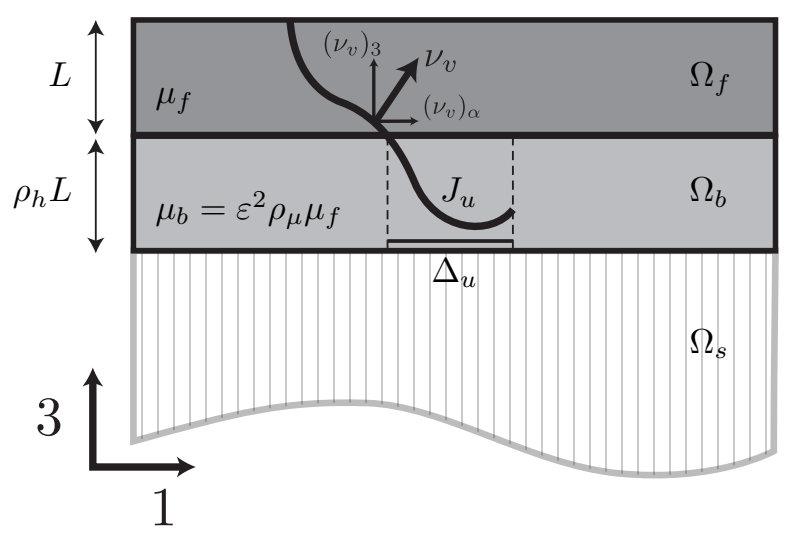

Figure 2: Rescaled brittle multilayer in scalar elasticity. Cracks are identified by the jump set $J_{u}$ of the admissible displacement field $u \in S B V\left(\Omega_{f} \cup \Omega_{b}\right)$. Thicknesses, stiffnesses and toughnesses verify a scaling law depending upon the small parameter $\varepsilon$.

Theorem 3.1. For any $u \in \mathcal{C}(\omega)$ let us define:

$$
\mathcal{E}_{0}(u):=\frac{L \mu_{f}}{2} \int_{\omega}\left|\nabla^{\prime} u-\epsilon_{0}^{\prime}\right|^{2} d x^{\prime}+\frac{L \mu_{b}}{2 h_{f} h_{b}} \int_{\omega \backslash \Delta_{u}}|u-w|^{2} d x^{\prime}+L G_{f} \mathcal{H}^{1}\left(J_{u}^{\prime}\right)+\frac{L G_{b}}{h_{f}} \mathcal{H}^{2}\left(\Delta_{u}\right),
$$

where

$$
\Delta_{u}:=\left\{x^{\prime} \in \omega:\left|u\left(x^{\prime}\right)-w\left(x^{\prime}\right)\right|>u_{d}:=\sqrt{\frac{2 G_{b} h_{b}}{\mu_{b}}}\right\}
$$

is the delamination set. Then the energy $\mathcal{E}_{0}$ admits at least one minimizer over $\mathcal{C}(\omega)$ and

$$
\min _{u \in \mathcal{C}(\omega)} \mathcal{E}_{0}(u)=\lim _{\varepsilon \rightarrow 0} \min _{u \in \mathcal{C}_{w}(\Omega)} \mathcal{E}_{\varepsilon}(u)
$$

In addition, if $u_{\varepsilon}$ is a minimizer of $\mathcal{E}_{\varepsilon}$ over $\mathcal{C}_{w}(\Omega)$, and $u_{\varepsilon} \rightarrow u_{0}$ strongly in $L^{2}\left(\Omega_{f}\right)$ for some $u_{0} \in \mathcal{C}(\omega)$, then $u_{0}$ is a minimizer of $\mathcal{E}_{0}$ over $\mathcal{C}(\omega)$.

The energy (14) of the limit model can be mechanically interpreted as the energy of a membrane on an elastic foundation à la Wrinkler undergoing in-plane displacements $u \in \mathcal{C}(\omega)$. The fracture energies naturally discriminate transverse cracks $J_{u}^{\prime}$ and debonded regions $\Delta_{u}$, the former being of codimension 1 while the latter are of codimension 0 in the two-dimensional limit domain $\omega$. The debonded regions are explicitly determined by the local threshold criterion (15) on the absolute value of the mismatch between the membrane displacement $u$ and the imposed displacement $w$ on the substrate. The elastic energy density comprises a contribution $\frac{L \mu_{f}}{2}\left|\nabla^{\prime} u-\epsilon_{0}^{\prime}\right|^{2}$, a membrane energy, estimating the elastic energy in the film; and a contribution $\frac{L \mu_{b}}{2}|u-w|^{2} /\left(h_{f} h_{b}\right)$ due to the interaction with the substrate, estimating the elastic energy in the bonding layer. The latter contribution is present only in bonded regions $\omega \backslash \Delta_{u}$

The proof of Theorem 3.1 is based on a $\Gamma$-convergence approach, and its structure is rather classical in dimensional reduction. It rests on three lemmas:

1. Compactness: If $\left(u_{\varepsilon}\right)$ is a sequence with uniformly bounded energy $\mathcal{E}_{\varepsilon}$, then (up to a subsequence) it converges strongly in $L^{2}\left(\Omega_{f}\right)$ to some $u \in \mathcal{C}(\omega)$;

2. Lower bound: For any $u \in \mathcal{C}_{w}(\omega)$ and for any sequence $\left(u_{\varepsilon}\right) \subset \mathcal{C}_{w}(\Omega)$ such that $u_{\varepsilon} \rightarrow u$ strongly in $L^{2}\left(\Omega_{f}\right)$, then

$$
\mathcal{E}_{0}(u) \leq \liminf _{\varepsilon \rightarrow 0} \mathcal{E}_{\varepsilon}\left(u_{\varepsilon}\right)
$$


3. Upper bound (existence of a recovery sequence): For any $u \in \mathcal{C}(\omega)$, there exists a sequence $\left(\bar{u}_{\varepsilon}\right) \subset \mathcal{C}_{w}(\Omega)$ such that $\bar{u}_{\varepsilon} \rightarrow u$ strongly in $L^{2}\left(\Omega_{f}\right)$ and

$$
\mathcal{E}_{0}(u) \geq \limsup _{\varepsilon \rightarrow 0} \mathcal{E}_{\varepsilon}\left(\bar{u}_{\varepsilon}\right) .
$$

The three previous properties ensure the convergence of minimizers as well as the convergence of the minimal value of the energy. Indeed, the compactness property implies that, if $u_{\varepsilon}$ is a minimizer of $\mathcal{E}_{\varepsilon}$ over $\mathcal{C}_{w}(\Omega)$, then a suitable subsequence converges strongly in $L^{2}\left(\Omega_{f}\right)$ to some $u_{0} \in \mathcal{C}(\omega)$, and the lower bound gives:

$$
\mathcal{E}_{0}\left(u_{0}\right) \leq \liminf _{\varepsilon \rightarrow 0} \mathcal{E}_{\varepsilon}\left(u_{\varepsilon}\right) .
$$

On the other hand, if $v \in \mathcal{C}(\omega)$ is a competitor for the reduced two-dimensional problem, the upper bound gives in turn the existence of some recovery sequence $\left(\bar{v}_{\varepsilon}\right) \subset \mathcal{C}_{w}(\Omega)$ converging strongly in $L^{2}\left(\Omega_{f}\right)$ to $v$, and such that:

$$
\mathcal{E}_{0}(v) \geq \limsup _{\varepsilon \rightarrow 0} \mathcal{E}_{\varepsilon}\left(\bar{v}_{\varepsilon}\right)
$$

According to the minimality property of $u_{\varepsilon}$ at fixed $\varepsilon$, we infer that:

$$
\mathcal{E}_{0}\left(u_{0}\right) \leq \liminf _{\varepsilon \rightarrow 0} \mathcal{E}_{\varepsilon}\left(u_{\varepsilon}\right) \leq \limsup _{\varepsilon \rightarrow 0} \mathcal{E}_{\varepsilon}\left(u_{\varepsilon}\right) \leq \limsup _{\varepsilon \rightarrow 0} \mathcal{E}_{\varepsilon}\left(\bar{v}_{\varepsilon}\right) \leq \mathcal{E}_{0}(v),
$$

which ensures that $u_{0}$ is a minimizer of $\mathcal{E}_{0}$ over $\mathcal{C}(\omega)$. Taking in particular $v=u_{0}$ in the previous chains of inequalities yields:

$$
\min _{u \in \mathcal{C}_{w}(\Omega)} \mathcal{E}_{\varepsilon}(u)=\mathcal{E}_{\varepsilon}\left(u_{\varepsilon}\right) \rightarrow \mathcal{E}_{0}\left(u_{0}\right)=\min _{u \in \mathcal{C}(\omega)} \mathcal{E}_{0}(u)
$$

which gives the convergence of the minimal value.

For the sake of conciseness and in order not to distract the readers not interested in the mathematical developments, we postpone the rigorous proofs of the above three lemmas to Appendix B. We report below some comments on the key features of the limit behavior of the system that are revealed by the mathematical analysis.

- Requiring the energy of the three-dimensional model (12) to be bounded as $\varepsilon \rightarrow 0$ implies that the terms $\partial_{3} u$ and $\nu_{u 3}$ must vanish in the film $\Omega_{f}$. Hence, in the limit $\varepsilon \rightarrow 0$ the displacements are expected to be constant through the thickness of the film, and the cracks in the film are purely transverse and span its whole thickness. This reasoning is made rigorous in the proof of the compactness property in Section Appendix B.1.

- The scaling hypotheses of Section 2.5 imply that the energy contributions associated to in-plane deformations in the film, through-the-thickness shear in the bonding layer, transverse cracks in the film, and in-plane cracks in the bonding layer are of the same order in $\varepsilon$ in (12). This entails the emergence of an interesting coupled problem involving all these phenomena, which is the one caught by the limit energy (14).

- In the energy (12) the elastic energy density associated to the in-plane gradient of the displacement inside the bonding layer $\Omega_{b}$ is proportional to $\varepsilon^{2}$, and thus vanishing for $\varepsilon \rightarrow 0$. This fact implies that in the limit $\varepsilon \rightarrow 0$ these gradients may possibly diverge. From a mathematical point of view, it translates into a lack of compactness inside the bonding layer. However, the displacement within the bonding layer is controlled by the imposed displacement on the interface with the substrate (Dirichlet boundary condition) and by the displacement of the film thanks to the continuity at the interface.

- The recovery sequence used in the proof of the upper bound (17) gives a deeper insight on the way in which the limit two-dimensional model approximates the three-dimensional system. Given a membrane displacement $u \in \mathcal{C}(\omega)$, this recovery sequence gives an optimal displacement field defined on the threedimensional domain $\Omega$ to minimize the total energy $\mathcal{E}_{\varepsilon}$ in the limit $\varepsilon \rightarrow 0$. Its full expression is given 
in (B.10). Figure 3(b) sketches the through the thickness distribution of this displacement field and the associated crack sets. In bonded regions, displacements are constant through the thickness of the film and affine within the bonding layer, varying from the boundary condition on $\Sigma$ to the value of the displacement of the film. In debonded regions no compatibility between the substrate and the film is enforced, hence the film is free to accommodate the inelastic strain.

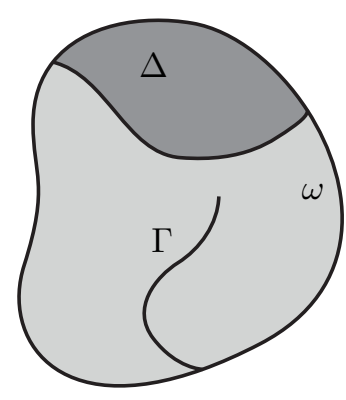

(a) Top view

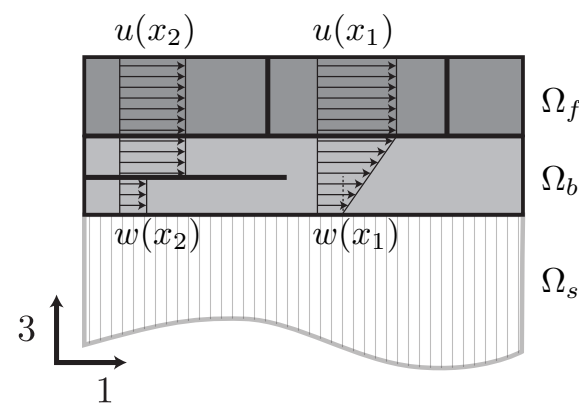

(b) Profile of the optimal displacement (recovery sequence)

Figure 3: In the limit two-dimensional body $\omega$, transverse cracks $\Gamma$ and debonded regions $\Delta$ are naturally discriminated, as shown in subfigure (a). Their nucleation and evolution under imposed loads is ruled by the variational principle. The recovery sequence used in the $\Gamma$-convergence giving an optimal through-the-thickness distribution of the displacement field is sketched in subfigure $3(\mathrm{~b})$ at points in bonded $\left(x_{1}\right)$ and debonded $\left(x_{2}\right)$ regions.

\section{Reduced model for two-dimensional vectorial elasticity}

The results of the previous section concern the case of scalar elasticity. Obviously, the physically relevant setting is that of vectorial elasticity, in which the displacement field is a vector. In this case, the rigorous asymptotic deduction of the limit two-dimensional brittle model involves further technical difficulties from the mathematical point of view. Here we proceed by induction and transpose (without proof) the results of the preceding section in the vectorial setting. In particular, we retain the form of the limit elastic energy, as the sum of the membrane energy of the film and the elastic foundation estimating the energy of the bonding layer; the geometric characterization of the crack surfaces and of the surface energy, naturally decoupling the two fracture modes, as the sum of the contributions due to transverse fracture and debonding. Currently, we are able to provide a full proof (not reported here) of this limit model only in the purely elastic case without fractures. As in Section 2, we formulate the problem in a strong form, i.e. without resorting to the use of functional spaces which allow for arbitrary discontinuities (the space $S B D$ of special functions of bounded deformation, the vectorial generalization of $S B V$ ). In the present context, this choice is mainly formal because we do not report on the mathematical analysis in the vectorial case.

\subsection{The reduced limit energy in two-dimensional elasticity}

We consider a two-dimensional brittle elastic membrane occupying the domain $\omega \subset \mathbb{R}^{2}$. In analogy to the scalar case, we discriminate between transverse cracks $\Gamma$ and debonded regions $\Delta$. In two dimensions, the former are a subdomain of $\omega$ of codimension 1 whereas the latter are a subdomain of codimension 0 . We assume that the membrane undergoes only in-plane displacements $u=\left(u_{1}, u_{2}\right)$ and that the displacement field is regular on the crack-free domain $\omega \backslash \Gamma$. The space of admissible displacements is:

$$
H^{1}\left(\omega \backslash \Gamma ; \mathbb{R}^{2}\right)
$$


In analogy with the reduced elastic energy (14) for the scalar case, given the loading as an inelastic isotropic strain $\epsilon_{0} \in L^{2}\left(\omega ; \mathbb{R}^{2 \times 2}\right)$ and an imposed displacement of the substrate $w \in H^{1}\left(\omega ; \mathbb{R}^{2}\right) \cap L^{\infty}\left(\omega ; \mathbb{R}^{2}\right)$, the elastic energy of the two-dimensional limit model for the vectorial case is taken as follows:

$$
P(u, \Gamma, \Delta):=\frac{1}{2} \int_{\omega \backslash \Gamma} A\left(\epsilon(u)-\epsilon_{0}\right) \cdot\left(\epsilon(u)-\epsilon_{0}\right) \mathrm{d} x+\frac{1}{2} \int_{\omega \backslash \Delta} \frac{\mu_{b}}{h_{b}}|u-w|^{2} d x
$$

where $A$ is the fourth-order tensor representing the isotropic stress-strain relation for the film as a twodimensional membrane in plane-strain. It is defined by:

$$
A \epsilon:=h_{f} \frac{\lambda_{f} \mu_{f}}{\lambda_{f}+2 \mu_{f}}(\operatorname{tr} \epsilon) I_{2}+2 \mu_{f} h_{f} \epsilon
$$

where $I_{2}$ is the two-dimensional identity tensor. The second integral in the potential energy (19) accounts for the presence of the bonding layer as an elastic foundation. The surface energy is assumed to be the sum of the contributions given by the transverse cracks $\Gamma$ and the debonded regions $\Delta$ :

$$
S(\Gamma, \Delta):=h_{f} G_{f} \mathcal{H}^{1}(\Gamma)+G_{b} \mathcal{H}^{2}(\Delta) .
$$

Hence, the total energy of the two dimensional model is:

$$
E(u, \Gamma, \Delta):=P(u, \Gamma, \Delta)+S(\Gamma, \Delta) .
$$

Remark 4.1. Equation (19) is the energy of a linear elastic prestressed plate undergoing purely in-plane displacements plus the energy of a linear elastic foundation in the bonded regions. In the purely elastic case (i.e. when $\Gamma=\Delta=\emptyset$ ), for the scaling hypotheses (9) and the assumed in-plane loading, the elastic energy (19) can be obtained as an asymptotic limit for $\varepsilon \rightarrow 0$ of the elastic energy of the three dimensional system introduced in Section 2. The problem is much more difficult in the brittle case because of the technicalities related to the handling of vectorial fields in free discontinuity problems.

\subsection{Nondimensionalization and free parameters}

Introducing the non-dimensional space variable and displacement field defined by

$$
x^{*}=x / x_{0}, \quad u^{*}=\frac{u-w}{\sqrt{G_{f} x_{0} / \mu_{f}}},
$$

the total energy (22) may be rewritten in the following non-dimensional form:

$$
\begin{aligned}
E^{*}\left(u^{*}, \Gamma^{*}, \Delta^{*}\right)=\frac{1}{2} \int_{\omega^{*} \backslash \Gamma^{*}} A^{*}\left(\epsilon^{*}\left(u^{*}\right)-\epsilon_{0}^{*}\right) \cdot\left(\epsilon^{*}\left(u^{*}\right)-\epsilon_{0}^{*}\right) \mathrm{d} x^{*}+\frac{1}{2} \int_{\omega^{*} \backslash \Delta^{*}} \kappa\left|u^{*}\right|^{2} \mathrm{~d} x^{*} \\
+\mathcal{H}^{1}\left(\Gamma^{*}\right)+\gamma \mathcal{H}^{2}\left(\Delta^{*}\right),
\end{aligned}
$$

where

$$
E^{*}=\frac{E}{h_{f} G_{f} x_{0}}, \quad A^{*}=\frac{A}{\mu_{f} h_{f}}, \quad \epsilon^{*}=\sqrt{\frac{\mu_{f} x_{0}}{G_{f}}} \epsilon, \quad \epsilon_{0}^{*}=\sqrt{\frac{\mu_{f} x_{0}}{G_{f}}}\left(\epsilon_{0}+\epsilon(w)\right)
$$

and

$$
\kappa=\frac{\mu_{b}}{\mu_{f}} \frac{x_{0}^{2}}{h_{f} h_{b}}, \quad \gamma=\frac{G_{b}}{G_{f}} \frac{x_{0}}{h_{f}} .
$$

Henceforth we consider the total energy in this form dropping the superscripted ${ }^{*}$ for the sake of conciseness. We conclude from this dimensional analysis that the non-dimensional parameters that fully characterize the energy are: 
- The loading parameter $\epsilon_{0}^{*}$, which absorbs the imposed displacement of the substrate and models both loading modes;

- The relative stiffness of the bonding layer and the film $\kappa$;

- The debonding to transverse cracking relative fracture toughness $\gamma$;

- The Poisson ratio $\nu_{f}$ of the film, that uniquely identifies the non-dimensional stiffness tensor $A^{*}$.

Note that one can always choose the scaling length $x_{0}=\sqrt{h_{f} h_{b} \mu_{f} / \mu_{b}}$ in order to have $\kappa=1$. However in that case the dimension of the domain (in $x_{0}$-units) will be an additional parameter. In the following we will adopt the opposite point of view, setting $x_{0}$ such that the diameter of the domain $\omega^{*}$ is 1 and keeping $\kappa$ as a free parameter. Note also that the competition between the membrane and the elastic foundation energies entails the existence of a non-dimensional internal characteristic length scale $\ell_{e}:=\kappa^{-1 / 2}$, measuring the decay of the elastic perturbations on the displacement field.

\subsection{Problem formulation}

We formulate below the static problem and the discrete-in-time evolution problem for the reduced model by extending those of Sections 2.3-2.4 to include the presence of the two fracture modes given by transverse and debonding cracks.

Problem 4 (Static solution of the reduced model). Given a loading $\epsilon_{0}$, find $u \in H^{1}\left(\omega \backslash \Gamma ; \mathbb{R}^{2}\right), \Gamma \subset \omega$, $\Delta \subseteq \omega$ such that

$$
E(u, \Gamma, \Delta) \leq E(\hat{u}, \hat{\Gamma}, \hat{\Delta}), \quad \forall \hat{\Gamma} \subset \omega, \forall \hat{\Delta} \subseteq \omega, \forall \hat{u} \in H^{1}\left(\omega \backslash \hat{\Gamma} ; \mathbb{R}^{2}\right) .
$$

This condition is equivalent to require that $(u, \Gamma, \Delta)$ solve the following minimization problem

$$
\inf \left\{E(u, \Gamma, \Delta): \quad \Gamma \subset \omega, \Delta \subseteq \omega, u \in H^{1}\left(\omega \backslash \hat{\Gamma} ; \mathbb{R}^{2}\right)\right\}
$$

Remark 4.2. As done in the case of scalar elasticity, for any admissible u, one can find explicitly the optimal debonded set by solving a linear optimization problem for the characteristic function $\chi_{\Delta}$ of the domain $\Delta$, see Theorem 3.1, which gives:

$$
\Delta_{u}:=\left\{x \in \omega:|u(x)|>u_{d}:=\sqrt{\frac{2 \gamma}{\kappa}}\right\} .
$$

Hence the static problem may be alternatively reformulated as the minimization of the energy

$$
E(u, \Gamma):=\int_{\omega \backslash \Gamma} \frac{1}{2} A\left(\epsilon(u(x))-\epsilon_{0}\right) \cdot\left(\epsilon(u)-\epsilon_{0}\right) \mathrm{d} x+\mathcal{H}^{1}(\Gamma)+\int_{\omega \backslash \Delta_{u}} \frac{\kappa}{2}|u|^{2} \mathrm{~d} x+\int_{\Delta_{u}} \gamma \mathrm{d} x
$$

In Equation (30), the energy density due to the film is a quadratic function of the the mismatch between the geometric strains $\epsilon(u)$ and inelastic strains $\epsilon_{0}$. On the other hand the energy density due to the bonding layer, say $f$, is quadratic in $u\left(f(u)=\kappa|u|^{2} / 2\right)$ before debonding $\left(|u| \leq u_{c}\right)$ and constant $(f(u)=\gamma)$ after debonding. Its dependence on $u$ is sketched in Figure 4. Even in the case without transverse cracks, the total elastic energy $E(u, \emptyset)$ is non-linear, non-smooth and non-convex with respect to $u$. As a consequence of the lack of convexity, we expect lack of uniqueness of the displacement solution as soon as debonding is triggered, even without considering transverse cracks. This problem has been studied is detail in the one-dimensional case in León Baldelli et al. (2013).

Problem 5 (Time-discrete evolution of the reduced model). Let $0=t_{0} \leq t_{1} \leq \ldots \leq t_{N}=T$ be the discretization of the time interval $[0, T]$ into $N$ time steps. A time-discrete quasi-static evolution for the displacement field and crack set of the reduced two-dimensional model is a mapping $t_{i} \mapsto\left(u^{i}, \Gamma^{i}, \Delta^{i}\right)$ that, given 


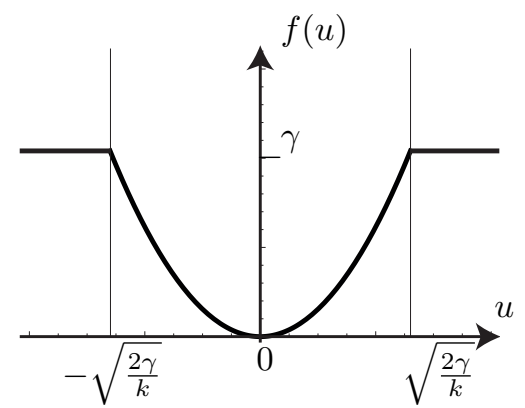

Figure 4: Qualitative properties of the energy density of the reduced model. The total energy density in the film is quadratic with respect to $u$ in the elastic phase and constant after debonding, see Eqn. (30).

the initial crack state $\left(\Gamma^{0}, \Delta^{0}\right)$ and the loading history $\epsilon_{0}^{i}$, verifies the following global unilateral minimality conditions $\forall i \in 1, \ldots, N$ :

$$
\begin{gathered}
\Gamma^{i} \supseteq \Gamma^{i-1}, \quad \Delta^{i} \supseteq \Delta^{i-1}, \\
E\left(u^{i}, \Gamma^{i}, \Delta^{i}\right) \leq E(\hat{u}, \hat{\Gamma}, \hat{\Delta}), \quad \forall \hat{\Gamma} \text { with } \Gamma^{i-1} \subseteq \hat{\Gamma} \subset \omega, \forall \hat{\Delta} \text { with } \Delta^{i-1} \subseteq \hat{\Delta} \subseteq \omega, \forall \hat{u} \in H^{1}\left(\omega \backslash \hat{\Gamma} ; \mathbb{R}^{2}\right) .
\end{gathered}
$$

These conditions are equivalent to require $\left(u^{i}, \Gamma^{i}, \Delta^{i}\right)$ to be a solution of the minimization problem

$$
\inf \left\{E(u, \Gamma, \Delta): \quad \Gamma^{i-1} \subseteq \Gamma \subset \omega, \Delta^{i-1} \subseteq \Delta \subseteq \omega, u \in H^{1}\left(\omega \backslash \Gamma ; \mathbb{R}^{2}\right)\right\} .
$$

\section{Regularized formulation and implementation}

This section details the numerical strategy followed to solve the quasi-static evolution problem for the reduced model presented in Section 4. It relies on the approximation by the means of elliptic functionals of the free discontinuity problem, as originally proposed in Ambrosio and Tortorelli (1990, 1992) for the Mumford-Shah functional (Mumford and Shah, 1989) in the field of image segmentation and exploited in Bourdin et al. (2008) in the framework of variational fracture mechanics.

\subsection{Regularized Formulation}

The solution of the quasi-static evolution of Problem 5 requires to minimize the energy with respect to the displacement field $u$, the debonded domain $\Delta$ and the crack set $\Gamma$, on which the displacement itself can be discontinuous. Resolving directly this kind of free-discontinuity problem is a major issue, because of the difficulty of the numerical treatment of the unknown crack set. The presence of the irreversibility condition, a unilateral constraint on the crack sets, further complicates the problem. As is now classical in variational fracture mechanics (Bourdin et al., 2008), we adopt here a regularized approach, in which the original problem is approximated by the minimization of a new functional where the transverse cracks $\Gamma$ are replaced by the localization of a smooth scalar field $\alpha(x): x \in \omega \rightarrow[0,1]$, taking the value 0 at sound points and 1 along cracks. The regularization of the energy functional (24) reads:

$$
\begin{aligned}
E_{\eta}(u, \alpha, \Delta):=\frac{1}{2} \int_{\omega} a(\alpha) A\left(\epsilon(u)-\epsilon_{0}\right) \cdot\left(\epsilon(u)-\epsilon_{0}\right) d x+ & \frac{1}{2} \int_{\omega} \kappa|u|^{2}\left(1-\chi_{\Delta}\right) d x \\
& +c_{w} \int_{\omega}\left(\frac{w(\alpha)}{\eta}+\eta\left|\nabla^{\prime} \alpha\right|^{2}\right) d x+\int_{\omega} \gamma_{\chi_{\Delta}} d x
\end{aligned}
$$

where $\chi_{\Delta}$ is the characteristic function of the debonded domain $\Delta, \eta$ is a scalar parameter,

$$
a(\alpha)=(1-\alpha)^{2}+k_{\eta}, \quad w(\alpha)=\alpha,
$$

and $c_{w}=1 /\left(4 \int_{0}^{1} \sqrt{w(\alpha)} d \alpha\right)=3 / 8$ is a normalization constant whose value is set to associate the transverse fracture energy of the Griffith model to a fully developed localization of the regularized field $\alpha$ (see Braides, 
1998; Pham et al., 2011a). In the expression (34) for $a(\alpha)$, the constant $k_{\eta} \ll \eta$ is a small residual stiffness required to ensure the regularity of the solutions when $\alpha$ reaches 1 .

The solution of the static problem formulated in the Problem 4 is approximated by

$$
\min \left\{E_{\eta}(u, \alpha, \Delta): \quad u \in H^{1}\left(\omega ; \mathbb{R}^{2}\right), \alpha \in H^{1}(\omega), 0 \leq \alpha \leq 1, \Delta \subseteq \omega\right\}
$$

For $\eta \rightarrow 0$ the solutions of (35) tend to the solutions of (24) in the sense of $\Gamma$-convergence ${ }^{1}$. This implies the term by term convergence of (33) to (24). In particular, the first integral of (33) approximates the elastic energy of the cracked film given by the first term of (24) and the second integral approximates the total transverse crack length given by the second term of (24). Note that regularization is performed only on transverse cracks $\Gamma$ since debonding cracks $\Delta$ are explicitely determined in the asymptotic process and do not induce discontinuities on the limit two-dimensional displacements. The advantages of using $w(\alpha)=\alpha$, instead of $w(\alpha)=\alpha^{2}$ as in Bourdin et al. (2000), are explained in some detail in Pham et al. (2011a). For quasi-static evolutions, the solutions of Problem 5 are approximated by formulating at each time step $t_{i}$ the constrained minimization problem:

$$
\min \left\{E_{\eta}(u, \alpha, \Delta): \quad u \in H^{1}\left(\omega ; \mathbb{R}^{2}\right), \alpha \in H^{1}(\omega), 0 \leq \alpha^{i-1} \leq \alpha \leq 1, \Delta^{i-1} \subseteq \Delta \subseteq \omega\right\}
$$

The proof of the convergence of the evolution problems is proved in Giacomini (2005) for the case of scalar elasticity, assuming that at each time one performs a global minimization of the regularized energy.

\subsection{Implementation}

Solving numerically the global minimization problem (36) for systems with a large number of degrees of freedom is not a viable option for the current state of the art in optimization methods. Motivated also by the physical considerations that will be detailed in the next subsection, we instead determine at each time $t_{i}$ a solution $u_{i} \in H^{1}\left(\omega ; \mathbb{R}^{2}\right), \alpha_{i}\left(\geq \alpha_{i-1}\right) \in H^{1}(\omega), \Delta^{i-1} \subseteq \Delta \subseteq \omega$ verifying only the associated first order (local) optimality conditions. Denoting by

$$
D_{f} F(f)(\hat{f}):=\left.\frac{d}{d h} F(f+h \hat{f})\right|_{h=0}
$$

the directional derivative of the functional $F$ with respect to the function $f$ in the direction $\hat{f}$, these conditions give the following system of coupled variational problems:

$$
\begin{array}{rrr}
u \text {-problem: } & D_{u} E_{\eta}(u, \alpha, \Delta)(\hat{u})=0, & \forall \hat{u} \in H^{1}\left(\omega ; \mathbb{R}^{2}\right) \\
\Delta \text {-problem: } & D_{\Delta} E_{\eta}(u, \alpha, \Delta)(\hat{\Delta}-\Delta) \geq 0, & \forall \hat{\Delta} \supseteq \Delta_{i-1} \\
\alpha \text {-problem: } & D_{\alpha} E_{\eta}(u, \alpha, \Delta)(\hat{\alpha}-\alpha) \geq 0, & \forall \hat{\alpha} \in H^{1}(\omega), \hat{\alpha} \geq \alpha_{i-1}
\end{array}
$$

where

$$
\begin{aligned}
D_{u} E_{\eta}(u, \alpha, \Delta)(\hat{u}) & =\int_{\omega}\left(a(\alpha) A\left(\epsilon(u)-\epsilon_{0}\right) \cdot \epsilon(\hat{u})+\kappa u \cdot \hat{u}\left(1-\chi_{\Delta}\right)\right) d x \\
D_{\Delta} E_{\eta}(u, \alpha, \Delta)(\hat{\Delta}) & =\int_{\omega}\left(\gamma-\frac{\kappa}{2}|u|^{2}\right) \chi_{\hat{\Delta}} d x \\
D_{\alpha} E_{\eta}(u, \alpha, \Delta)(\hat{\alpha}) & =\int_{\omega}\left(\left(\frac{d a}{d \alpha}(\alpha) A\left(\epsilon(u)-\epsilon_{0}\right) \cdot\left(\epsilon(u)-\epsilon_{0}\right)+\frac{c_{w}}{\eta} \frac{d w}{d \alpha}(\alpha)\right) \hat{\alpha}+c_{w} \eta \nabla \alpha \cdot \nabla \hat{\alpha}\right) d x
\end{aligned}
$$

\footnotetext{
1 The rigorous proof of this statement is omitted in the present paper for the sake of conciseness. The convergence without substrate energy $(\Delta=\emptyset, \kappa=0)$ and $w(\alpha)=\alpha^{2}$ is proven in Chambolle (2004). The statement can be trivially adapted to the case $\Delta \neq \emptyset, \kappa \neq 0$ ) observing that the additional terms are nothing but a continuous perturbation of the functional considered in Chambolle (2004) with respect to which the $\Gamma$-convergence (see e.g. Braides (1998)). The extension to more general energies including the case $w(\alpha)=\alpha$ is done in Braides (1998) for scalar elasticity and can be generalized without major issues to vectorial elasticity. Note also that, up to the debonding effect, the energy functional (24) is equivalent (at fixed elasticity) to a vectorial Mumford-Shah functional Mumford and Shah (1989), where the role of the "fidelity term" is played here by the elastic foundation.
} 
To solve this system at each time-step we extend to the present three-field case the alternate minimizations algorithm proposed by Bourdin et al. (2000). We solve iteratively each subproblem with respect to the corresponding field, leaving the other two fixed to the previously available values. More precisely we first solve in this way the $u-\Delta$ subproblem until convergence at fixed $\alpha$ and then iterate solving the $\alpha$-problem (see Algorithm 1). The $u$ problem at fixed $\alpha$ and $\Delta$ is a linear variational equation, which, after spacediscretization, we solve using standard iterative Krylov Subspace Solvers. Considering the irreversibility condition on the debonding set, the condition (38b) simply gives $\chi_{\Delta}(x)=1$ if the displacement passes a given threshold at the point $x$. On the other hand, the $\alpha$-problem at fixed $u$ and $\Delta$ is a linear variational inequality, which we solve using the bound-constrained Newton Trust-Region solver provided in the optimization toolbox TAO (Munson et al., 2012). Parallel data representation and linear algebra are based on the PETSc toolkit (Balay et al., 2012). On the other hand the solution of the problem (38b) at fixed $u$ is explicit and local in space.

We do not need any special treatment for the discretization of the computational domain. An unstructured conforming triangulation of the reference domain is obtained by a Delaunay algorithm and the discretization of the fields is done by standard triangular finite elements of class $\mathcal{P}^{1}$ on the fixed mesh. The discrete fields are subscripted by an $h$ referring to the average diameter of the triangulation. The parameter $\eta$ controls the width of the localization band of the fracture field, which is of the same order of magnitude of $\eta$. The computational mesh is uniformly fine (the mesh is such that $h \ll \eta$ ) in order to capture and represent the steep gradients within the localization band. A coarse mesh produces a systematic overestimation of the dissipated surface energy.

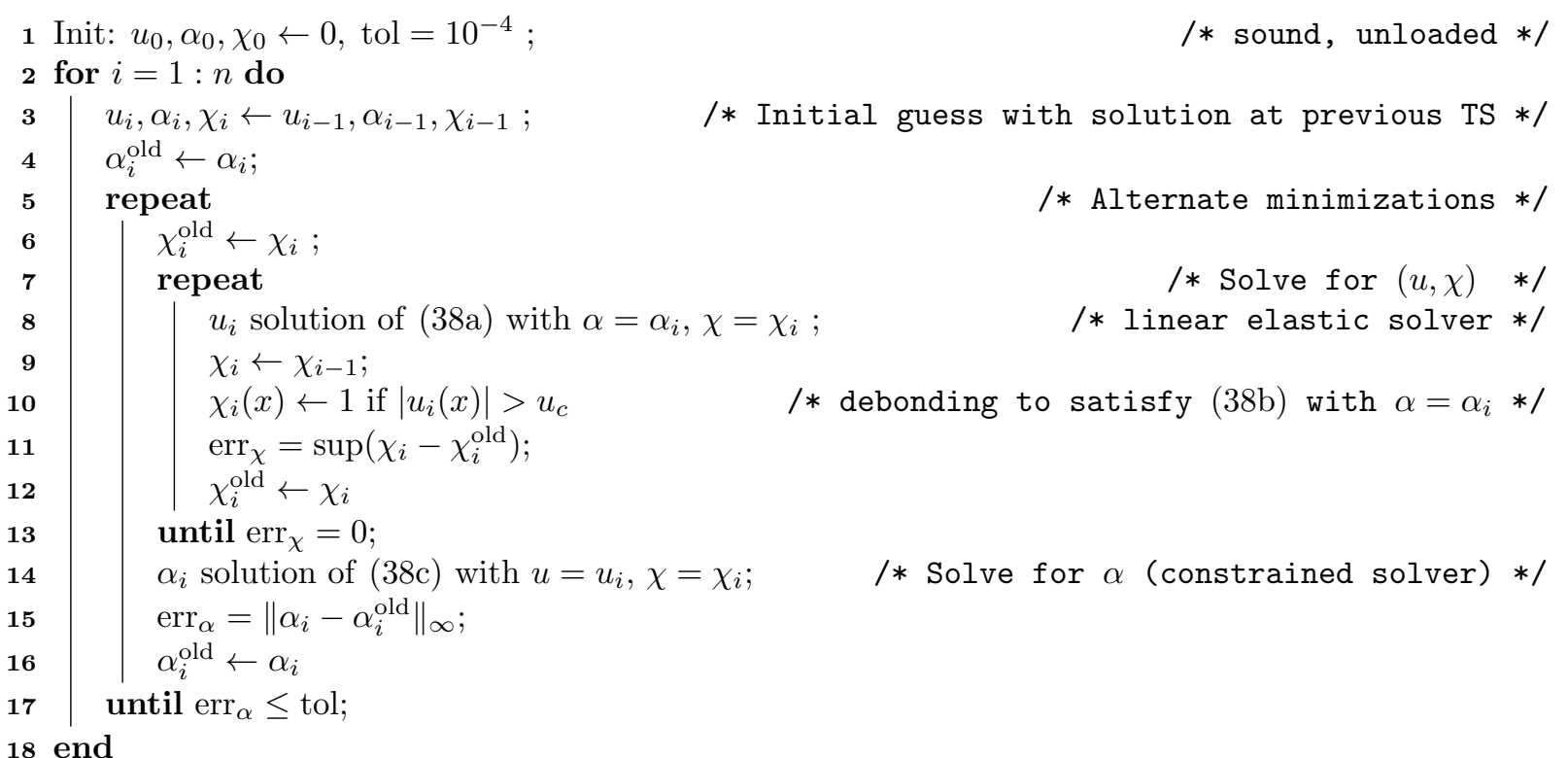

Algorithm 1: Algorithm for the solution of the quasi-static time-discrete evolution problem with transverse fracture and debonding. At each minimization in $(u, \chi)$ and $\alpha$ are performed at each time step, until convergence. For the sake of conciseness, we replace here $\chi_{\Delta}$ by $\chi$.

\subsection{Mechanical interpretation of the regularized model with local minimization}

The regularized energy (33) falls within the class of the Ambrosio-Tortorelli approximations of free discontinuity problems and is an instance of the gradient damage functional studied in (Pham et al., 2011a,b; Pham and Marigo, 2012). Indeed, the functions $w(\alpha)$ and $a(\alpha)$, besides satisfying the hypotheses underlying the $\Gamma$-convergence result (see Braides (1998)), verify the additional constitutive assumptions that allow us to identify $a(\alpha)$ as a stiffness function, $w(\alpha)$ as a dissipation function, and $\alpha$ as a damage field (Pham et al., 2011b). In this framework, the parameter $\eta$ becomes the internal characteristic length of the damage model, 
and it has to be thought of as a material parameter. The evolutions associated to the computed solutions of (36), numerically obtained enforcing the first order necessary optimality conditions (38), are consistent with the notion of irreversible evolution of energetically stable states, i.e. of unilateral local minimizers of the total energy. In this sense, transitions between states take place in correspondence to the loss of stability of the current state. Although a study of the stability properties of the energy $E(u, \alpha, \Delta)$ of Equation (33) depending upon the parameters $(\kappa, \nu, \gamma, \eta)$ is beyond the scope of this work, we provide an interpretation of the critical loads in the one-dimensional traction test of a slender strip in Section 6.1. Denoting by $\sigma=a(\alpha) A\left(\epsilon(u)-\epsilon_{0}\right)$ the (dimensionless) stress tensor in the film, equation (38c) implies that an elastic state where $\alpha=0$ is admissible only if

$$
A^{-1} \sigma \cdot \sigma \leq \frac{3}{8 \eta\left(1+k_{\eta}\right)}
$$

The inequality above gives an explicit relation between the internal length $\eta$ and the elastic limit stress $\sigma_{c}$ in the film, showing that $\sigma_{c} \propto 1 / \sqrt{\eta}$.

\section{Numerical Experiments}

We perform three sets of numerical experiments to illustrate the capabilities of the formulation in simple cases. We focus on the cases of multiple cracking and possible debonding of a slender strip, of a disk and on cracking of a geometrically complex domain. The first set of experiments is also intended to verify the numerical code against the closed form solutions presented in León Baldelli et al. (2013). The second set of experiments shows the capability of capturing geometrically complex two-dimensional crack patterns. Lastly, the third experiment provides a qualitative comparison with a real-life example inspired by the multiple cracking of a vinyl lettering panel.

In what follows, we consider the systems loaded by an inelastic isotropic strain $\epsilon_{0}=t I_{2}$ increasing linearly with time.

\subsection{Multiple cracking and debonding of a slender strip}

We perform a set of verification experiments for the problem of multifissuration and delamination of a one-dimensional stiff film bonded to a substrate. Let us consider a slender brittle elastic body, its reference domain being $\omega:\left\{x \in\left[0, x_{0} L\right] \times\left[0, x_{0} a\right]\right\}$, with $a \ll L$. To get an exact reference solution, the problem may be conveniently approximated by the one-dimensional model considered in León Baldelli et al. (2013), provided that $a \ll \ell_{e}, \ell_{e}=\kappa^{-1 / 2}$ being the characteristic length of the elastic problem. The condition $a \ll \ell_{e}$ implies that the stress field, under an equi-biaxial imposed inelastic strain, is essentially uniaxial.

The computational domain is of unit length and height $a=2 \cdot 10^{-2}$, it consists of approximately $7 \cdot 10^{3}$ degrees of freedom. The average mesh size is $h=2 \cdot 10^{-3}$, the value of $\eta=2 \cdot 10^{-2}$ is held fixed for the three experiments, the ratio $\eta / h$ is 10 and the quasi-static simulation consider loading multipliers up to $T_{\max }=11$. Note that as long as $\eta \ll \ell_{e}$ no coupling arises at the length scale of $\eta$ between the damage localization bands and the elastic displacement field, which varies over a length scale of order $\ell_{e}$.

We perform numerical experiments based on the closed form evolutions reported in León Baldelli et al. (2013). The analytical computation in the latter work is obtained by a global minimization statement, whereas the numerically computed solutions presented here satisfy only first order local optimality conditions and may not be global minimizers.

Transverse fracture experiment. In Figure 5 we represent the outcome of a transverse fracture experiment. The non-dimensional parameters characterizing the experiment are: $\kappa=36.0$ and $\gamma=10 \cdot 10^{4}$. The chosen stiffness ratio $\kappa$ corresponds to an internal characteristic elastic length scale $\ell_{e}=1 / 6$, hence $\eta / \ell_{e}=0.12$. The sound elastic energy branch loses stability at $t=4.81$, see Figure $5(\mathrm{c})$, when the system jumps towards the cracked state with one transverse crack in the center of the domain. This releases elastic energy at the expense of the surface energy, as it can be seen in the energy chart in Figure 5(c). As the load increases further, the system undergoes the elastic loading phase of the two segments. At $t=7.46$ the loss of stability 
(a)
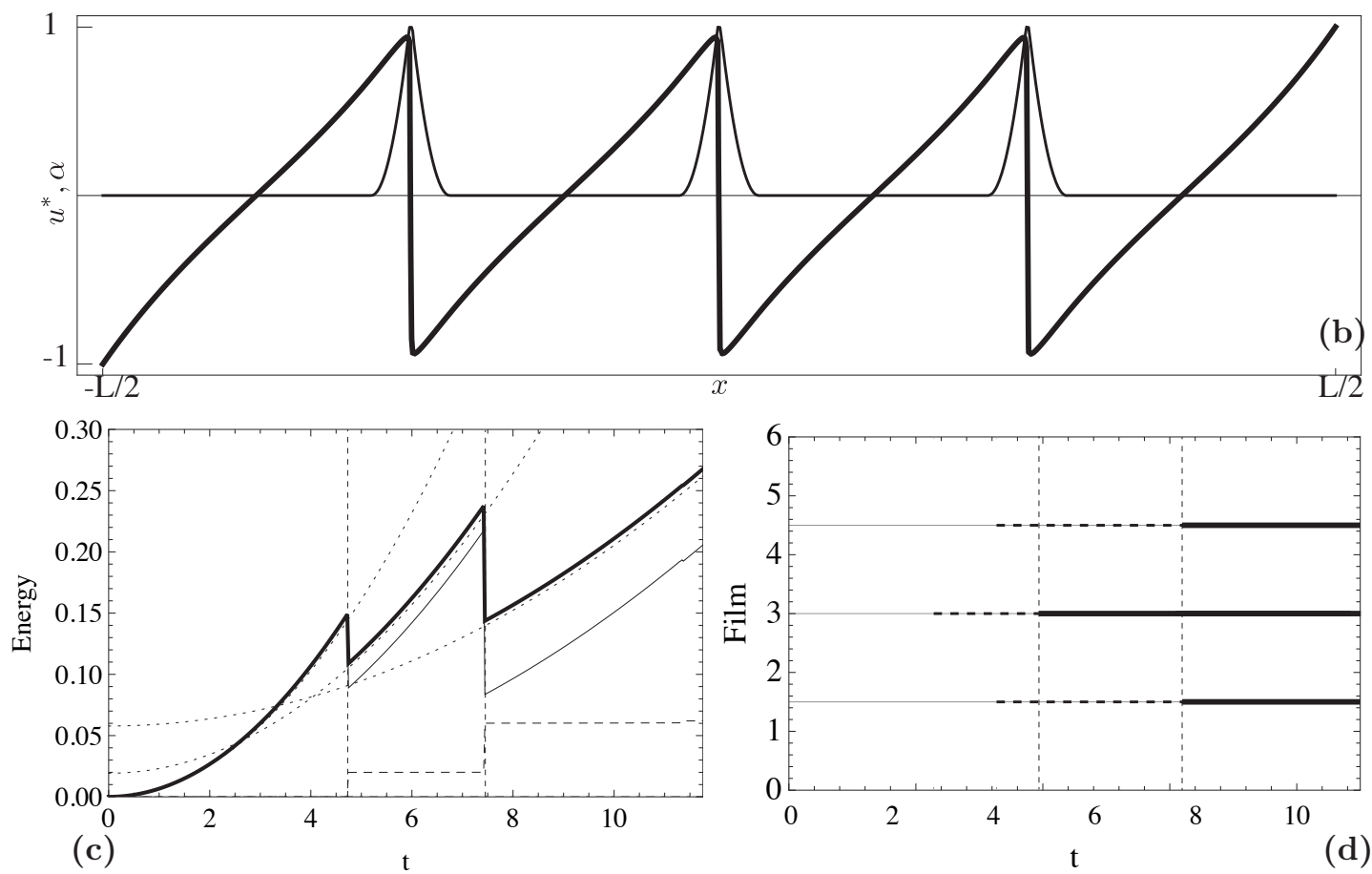

Figure 5: Top: snapshot of the fracture field at $t=T_{\max }$ for the perfectly bonded transverse fracture experiment. Cracks are equidistributed and represented by the localization of the damage field $\alpha$. The values of $\alpha \in[0,1]$ are mapped onto a "inverted-hot" color table, blue corresponding to $\alpha=0$ (sound material), red corresponding to $\alpha=1$ (fully developed fracture). Middle: displacement and fracture field along the axis $[-L / 2, L / 2] \times\{0\}$ for $t=T_{\max }$. The displacement field $u^{*}(x)=u(x) / \max _{x \in \omega} u(x)$ is normalized and displayed with a thick solid line. The fracture field $\alpha$ is shown with a thin black stroke. Bottom: in the energy chart (left) the total energy is plotted in bold line, the energy transverse fracture energy with a dashed line and the elastic energy with a thin solid line. Grid lines indicate the critic loads for transverse cracking. The total energy of the closed form solutions reported in León Baldelli et al. (2013) is plotted with a dotted line. In the space-time evolution diagram (right), the domain $\omega$ is represented on the vertical axis and the load on the horizontal axis. Solid black horizontal lines indicate the position of cracks during the evolution.

of this solution leads to the appearance of two add-cracks, each at the middle of the segments. The snapshot of the last loading step is shown in Figure 5(a) and the profile of the displacement and fracture fields are shown in Figure 5(b). The computed energy branches are seamlessly superposed to the analytical ones, and the evolution of the system is illustrated by the space-time chart in Figure 5(d). Critical times at which cracking happens differ between the numerical experience and the analytic computation, due to the global $v s$. local setting of minimization. As expected, the critical loads corresponding to the local minimization criterion systematically overestimate those satisfying the global criterion. 


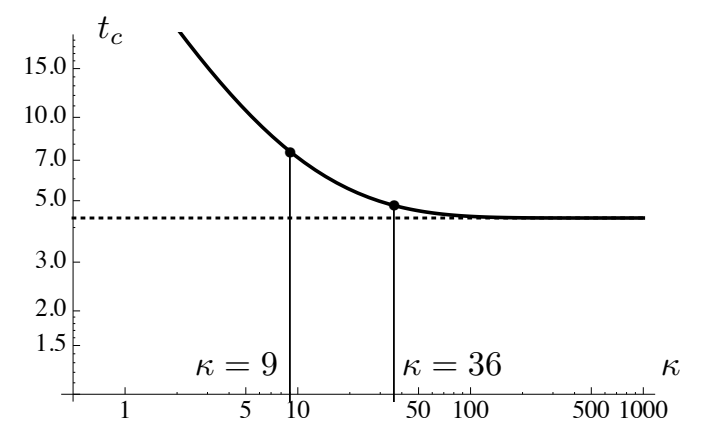

Figure 6: Critical loads of the transverse fracture experiments are compared to the elastic limit (Equation (40)) computed with the stability condition (38c) and plotted against the relative stiffness $\kappa$. The plot is for $\eta=0.02$. The asymptote $\kappa \rightarrow \infty$ corresponds to the limit case of a long film with homogeneous stress. For $\kappa \rightarrow 0$ the critic load $t_{c} \rightarrow \infty$, this corresponds to the limit case of system in which no energy is stored in the bonding layer and the film freely accommodates the inelastic strain.

The critical fracture loads are interpreted under the light of the considerations sketched in Section 5.3. Using a one-dimensional model, the critical loading for leaving the purely elastic regime may be established analytically, using Equation (40). Indeed, for the elastic solution $(\alpha=0)$, the stress $\sigma$ as a function of the loading may be easily computed analytically (see León Baldelli et al., 2013). Substituting this expression into Equation (40), one finds that purely elastic solutions are admissible for loadings not greater than

$$
t_{c}(\kappa, \eta):=\frac{\sqrt{3 / 8}}{\sqrt{\eta\left(1+k_{\eta}\right)}} \frac{1}{\left(1-\operatorname{sech}\left(\frac{\sqrt{\kappa}}{2}\right)\right)} .
$$

The critical time for the elastic solution is plotted in Figure 6.1 as a function of the stiffness ratio $\kappa$ for $\eta=0.02$. It is a monotonic function of $\kappa$ decreasing from $+\infty$ for $\kappa \rightarrow 0^{+}$to $\sqrt{3 / 8 \eta\left(1+k_{\eta}\right)}$ for $\kappa \rightarrow \infty$. In the same figure, we display with black dots the critical load captured by the numerical experiment. The first transverse fracture appears for the strip of stiffness ratio $\kappa=36.0$ for $t=4.81$. It creates two uncracked strips of half-length that, recalling the definition of $\kappa$, have an equivalent stiffness ratio $\kappa / 4=9$. Both these two strips further break into two parts at the second critical load $t=7.46$. Both critical loads coincide, within a small error, with the critical loads of the elastic solution given by equation (41) for $\kappa$ equal to 36 and 9, respectively (see Figure 6.1). Indeed, as done in (Pham et al., 2011b) for the case of a bar in traction, it may be shown that for sufficiently long strips the elastic limit also coincides with the stability limit of the solution without damage localizations (i.e. fractures). When passing this limit, the fundamental undamaged solution becomes unstable. The numerical algorithm based on alternate minimizations detects new descent directions and automatically jumps to a new (stable) solution branch, implying newly added cracks. Note that after the first transverse crack, the first order stability properties of the two cracked segments are almost insensitive of the half localizations at the boundaries. This does not hold asymptotically when inducing further fragmentations, upon increasing the load and producing small segments whose characteristic elastic length is comparable to the internal length $\eta$ associated to the damage localization. This regime is not explored in the present work, in all the experiments the internal length of the damage process $\eta$ is kept smaller than the elastic length $\ell_{e}=\kappa^{-1 / 2}$.

Debonding experiment. Figure 7 refers to a debonding experiment with the same equivalent stiffness $\kappa=$ 36.0 as the experiment above (and hence the same elastic length $\ell_{e}=1 / 6$ ) and a lower toughness ratio $\gamma=0.50$. The sound elastic bonded branch is followed by the debonding phase, whose onset is at $t=2.25$. Elastic energy is released at the expense of the debonding surface energy. The total energy asymptotically approaches the limit of the energy of the completely debonded film $E_{\infty}=L \gamma$. The computed energy coincide with the analytical energies and also the evolution are identical. In fact, differently from the perfectly bonded transverse cracking experiment, both in the numerical and closed form computations, the evolution of debonding relies only on first order optimality conditions León Baldelli et al. (2013). A snapshot of the last time step is displayed in Figure 7(a) and the displacement and debonding fields in Figure 7(b). 
(a)
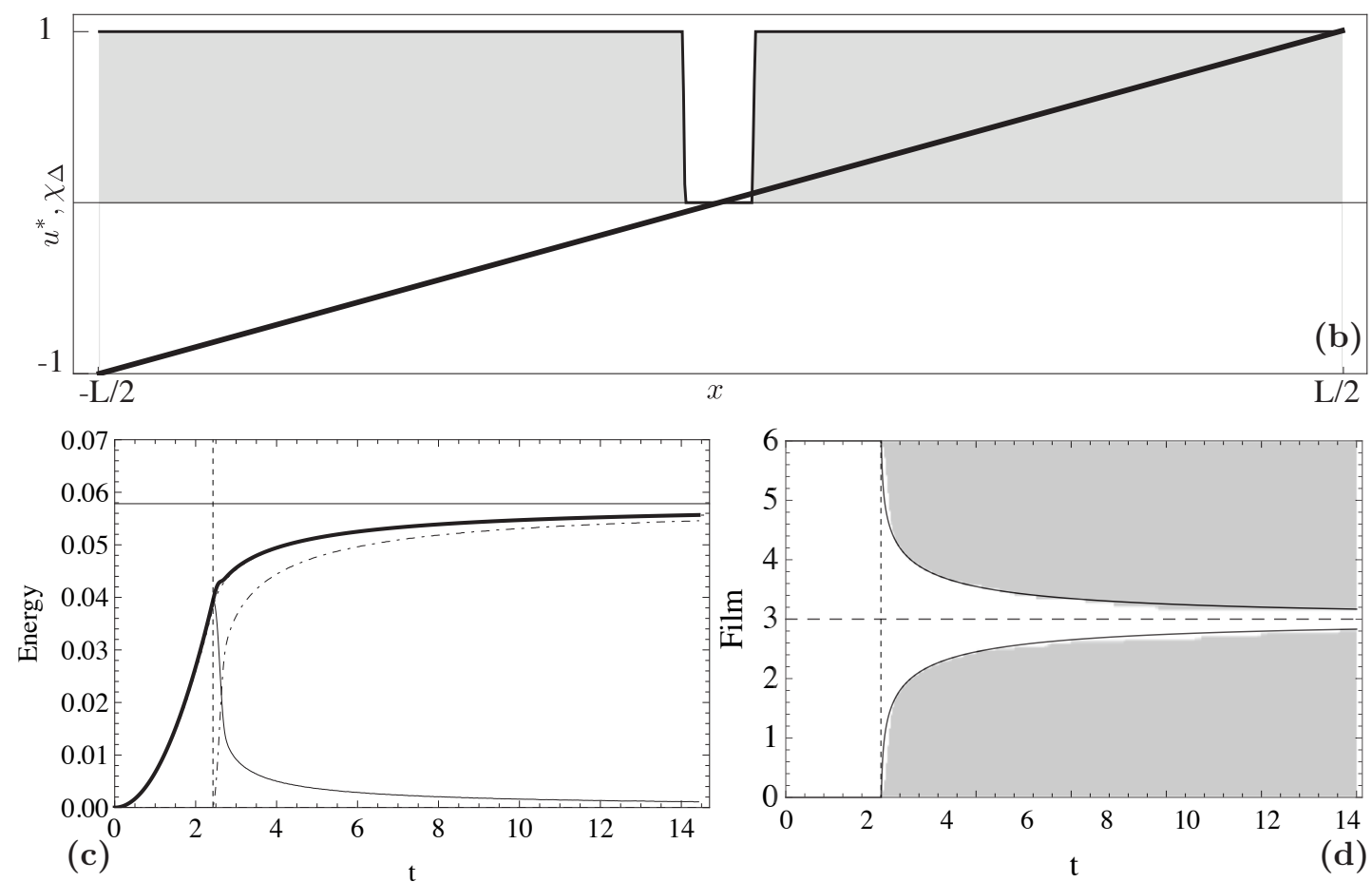

Figure 7: Top: Fracture and debonding fields at $t=T_{\max }$ for the deboonding experiment. Debonding $\left(\chi_{\Delta}(x)=1\right.$ is the darker area) is symmetric about the two axes. Middle: The characteristic function of the debonded domain is shaded gray, displacement is plotted with a thick stroke. Note that, in debonded regions, the displacement in linear and accommodates the imposed strain. Bottom: energy chart (left) and evolution diagram (right). Debonding onset and its evolution coincide in both numeric and analytic computations as they are derived as consequences of the first order necessary condition for energy optimality. The thin black line in the space-time evolution plot (right) is the analytical solution to the debonding problem obtained in León Baldelli et al. (2013).

The debonded domain is symmetric with respect to the axes of the film. In the debonded domain, the displacement is linear and accommodates the imposed inelastic strain, hence the energy vanishes. We remark that in spite of the lack of uniqueness of the displacement field in the debonded solution (recall that all states with equal debonded length have equal energy, irrespective of the location of the debonded area), numerical computations seem to favor symmetric solutions. The space-time chart illustrates the evolution, showing the bonded domain for a given load intensity.

Coupled experiments. Experiments in Figures 8 and 9 show the interplay between the two failure modes. In these two experiments, the system exhibits one (resp. three) transverse cracks prior to peripheral debonding. The evolutions are obtained choosing $\kappa=36.0\left(\ell_{e}=1 / 6\right)$ and $\gamma=2.2($ resp. $\kappa=64.0$ and $\gamma=3.1$, i.e. $\left.\ell_{e}=1 / 8\right)$. The corresponding energy chart and state diagrams are shown in Figures 8(c) and 9(c). A higher order effect is observed at the onset of debonding for the second coupled experiment due to the boundary layer induced by the fracture field around the middle crack causing local softening. This breaks the symmetry of the boundary conditions for the two segments. The effect is visible in the space-time evolution and in the debonding and elastic energy terms in Figure 9(d), although not noticeable at the global level of the total energy. 
(a)
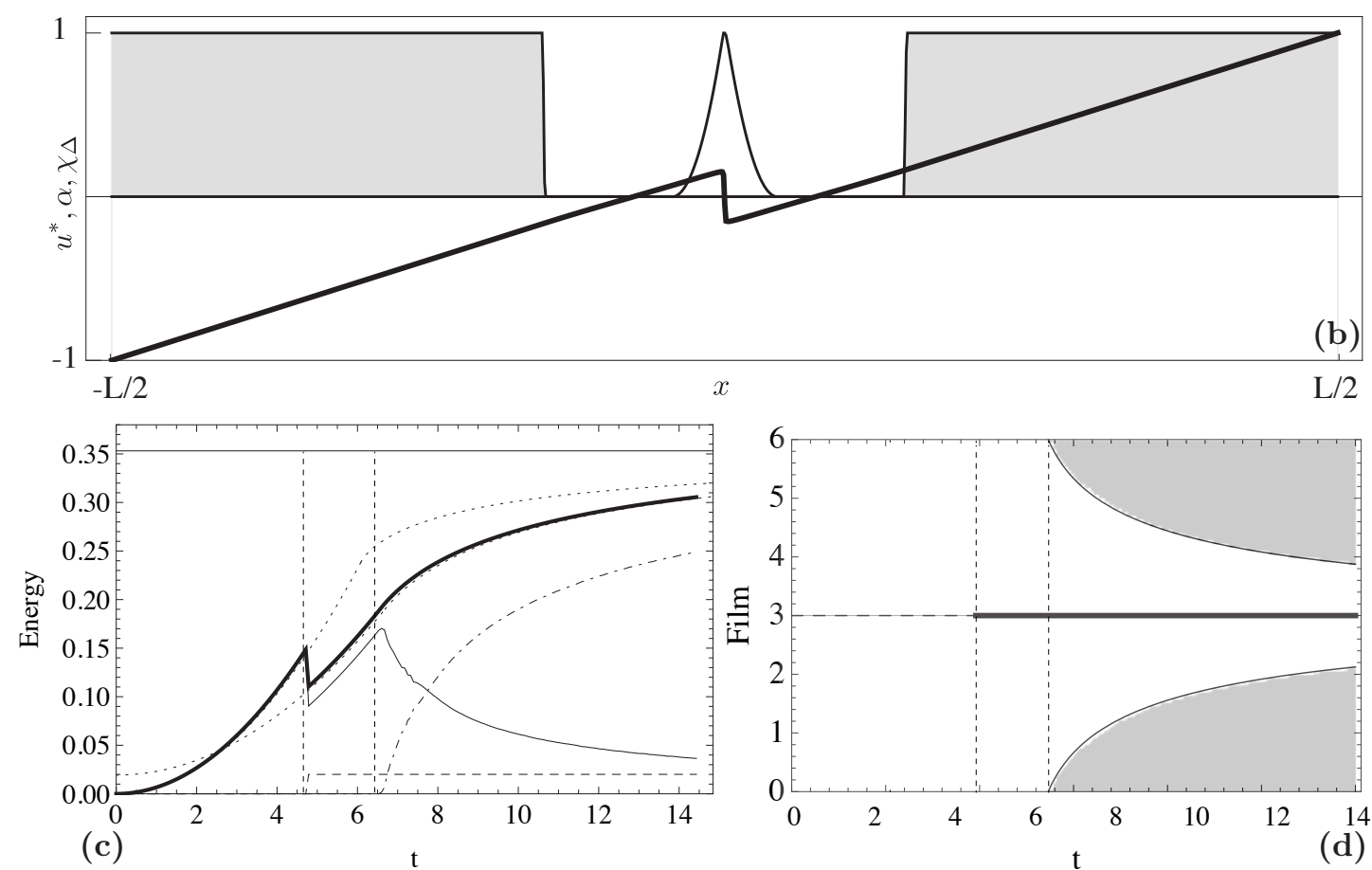

Figure 8: Top: fracture and debonding fields on the reference domain $\omega$ at $t=T_{\max }$ for the first coupled experiment; one transverse crack in the center and symmetric debonding starting from the boundaries. Middle: a single crack in the center of the film, the symmetric debonded region and the displacement field. Bottom: the analytic solution (global minimization) anticipates the appearance of the crack of the numerical experiment (local minimization). The debonding onset and evolution in both cases are equal.

\subsection{Multiple cracking and debonding of a thin disk}

We illustrate the ability to capture complex crack geometries and time-evolutions considering the problem of a homogeneously prestressed circular elastic wafer. We analyze qualitatively the outcome of the experiments showing its soundness on a mechanical basis and its coherence with the mechanical intuition and commonly reported experimental observations. The computational domain is of unit diameter, each experiment is univocally identified by four non-dimensional parameters: the relative stiffness $\kappa$, the relative toughness $\gamma$, the Poisson ratio $\nu$ and the maximum load intensity $T_{\max }$.

We introduce a non-homogeneity in order to explore more complex crack patterns around the sound elastic state. In the center of the wafer, we place a domain $\mathcal{D}_{\eta}$ of size of $\mathcal{O}(\eta)$ where we set $\alpha=1$, see Figure 11(a).

Multiple cracking only. The non-dimensional parameters for this experiment are $\kappa=200.0\left(\ell_{e}=0.071\right)$, $\gamma=4.6, \nu=0.3$ and $T_{\max }=3.76$. The wafer undergoes an elastic loading phase during which the domain $\omega \backslash \mathcal{D}_{\eta}$ remains sound. As the load increases, nucleation is localized in the neighborhood of the domain $\mathcal{D}_{\eta}$. Sudden fracture occurs at $t=2.0$ : a network of cracks of finite length appears in a single loading step and a network of hexagonal polygons forms. We observe a non-axisymmetric solution to a problem with axisymmetric data. Away from the boundaries, the cracks are structured in a network of six hexagons all with the same characteristic diameter. We capture the spontaneous nucleation of cracks within the domain, away from possible boundary non-homogeneities, with preference of $2 \pi / 3$-junctions over $\pi / 2$-junctions. This feature corresponds to regimes in which the sound solution is stable until load intensities high enough to 
(a)
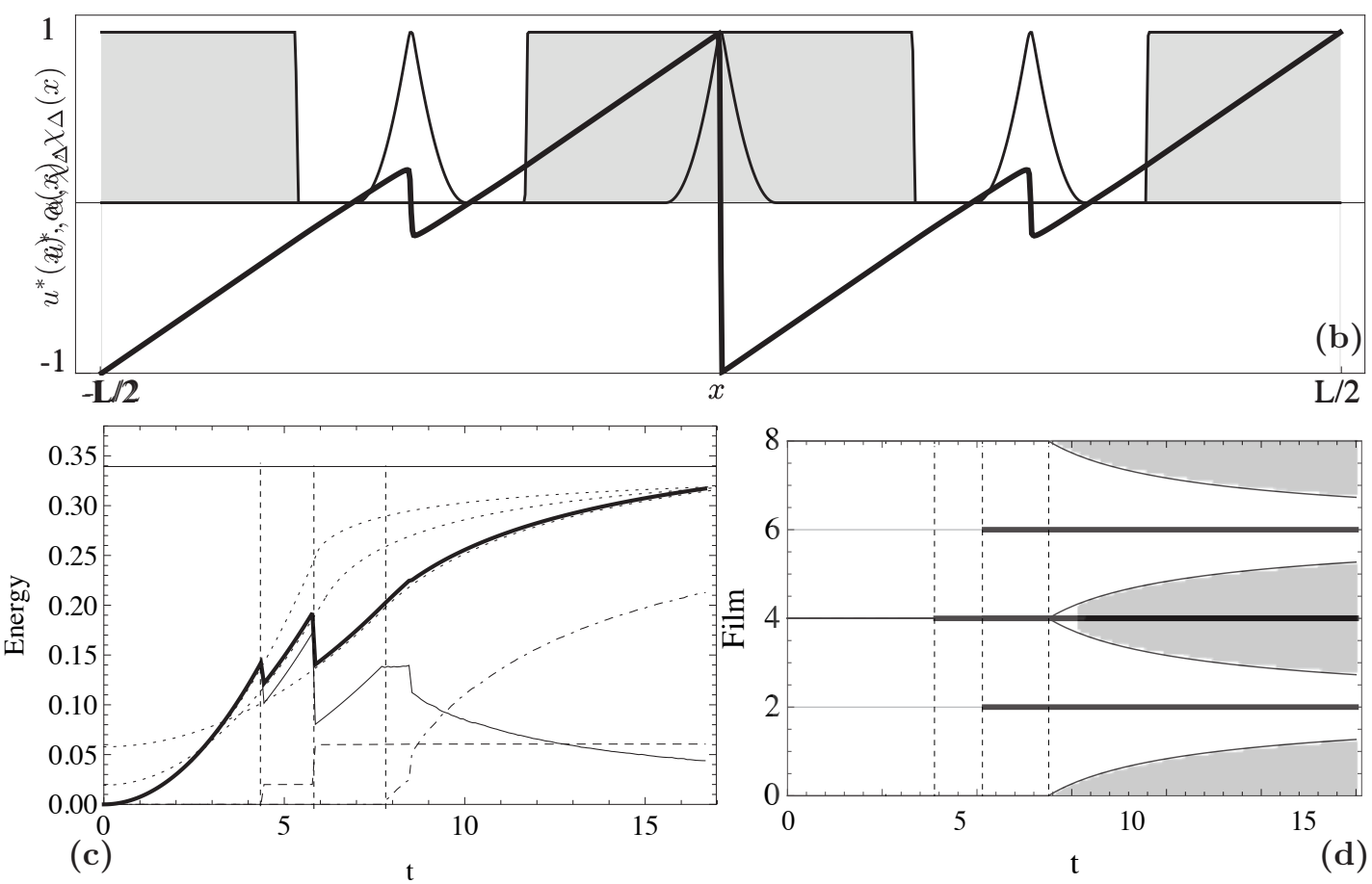

Figure 9: Top: fracture and debonding fields on the reference domain $\omega$ at $t=T_{\max }$ for the second coupled experiment; three equidistributed transverse cracks and debonding starting from the free boundaries of each segment. Middle: three equidistributed cracks (thin solid), debonded region (light gray shaded) and displacement field (thick solid). Bottom: energy chart and space-time evolution of the film. In (right) horizontal lines identify the position of the cracks: solid and dotted strokes refer to the numerical experiment and the analytic solution, respectively. Note the higher order effect due to softening at the onset of debonding at $x=4$.

release sufficient energy to pay for the creation of the network of cracks, which, in the numerical experiment, consists in six hexagons. Fracture patterns with $2 \pi / 3$-junctions are observed in the experiments of Groisman and Kaplan (1994); Santanach Carreras et al. (2007); Toga and Alaca (2006). More commonly, experiments on thin film fracture under isotropic and homogenous loads reports irregular mud cracks with $\pi / 2$-junctions. This may be due to a regime in which the material heterogeneities and imperfections dominate the nucleation phase letting a crack appear at lower load levels. In fact, in the cited experimental references, the predominance of $2 \pi / 3$-junctions is prominent in regimes where cracking is less likely, or equivalently, when cracking appears for high (dimensional) load intensities, that is, for systems in which the film's thickness is close to the so-called critical thickness $h_{c}$. The latter quantity identifies the thickness below which no cracking is observed in the experimental conditions. A shift, from $\pi / 2$ to $2 \pi / 3$, of the distribution of the joint angles, is reported in Groisman and Kaplan (1994) in the regimes where $h_{f}$ approaches $h_{c}$ and in Goehring et al. (2010) along cyclic loading tests which favour crack reorganization and maturation.

Note that when cracks intersecting free boundary form a right angle, instead of the $2 \pi / 3$ angles observed in the bulk of the domain. This is the case for all six intersections with the edge of the wafer. Indeed, under homogeneous loads and near a boundary, the direction of maximum stress is parallel to that boundary and elastic energy release per unit of crack length is maximized for crack advancing perpendicularly to it. As soon as cracks are close enough to be able to interact, they turn and produce the same phenomenology observed for the intersection of a crack with a free boundary. In Figures 11(b) and 11(c) we observe a turning crack in order to approach the existing crack at an angle of $\pi / 2$. 
As the load intensity increases further, new cracks are created by subdivision of the polygons. New isolated cracks appear forming joints at $\pi / 2$ with pre-existing cracks of free boundaries, and new $2 \pi / 3-$ joints form, see Figures 11(d)-11(f). The crack pattern becomes tighter with all polygons having the same characteristic size. As the polygons become smaller, new joints at sound points are less likely to appear and straight joints become predominant.

Multiple cracking and debonding. Reducing the toughness of the bonding layer to $\gamma=1.4$, all other conditions held fixed, highlights the interplay between transverse cracking and debonding. The evolution of the elastic sound phase and the first stages of cracking are the same as in the previous experiment. Since polygons have different sizes, unlike in the one-dimensional experiment, debonding does not appear simultaneously at the boundary of each polygon. At $t=2.5$ the largest cells start debonding from the outer boundary. As the load increases further, smaller polygons undergo debonding. At $t=T_{\max }$ all polygons have debonded and the typical diameter of the bonded regions is the same for all polygons. In this experiment, like in the one-dimensional slender strip, we observe a size effect due to the existence of an intrinsic characteristic length scale. The latter is revealed by the fracture processes. Indeed, the competition between the surface energies fixes the maximum diameter (at a given load) of the domain that can be completely bonded. This quantity is a decreasing function of the load and it determines a threshold distinguishing two phenomenologically different regimes: that of multiple cracking and that of extensive debonding. In fact, cracking will occur as long as the size of the subdomains identified by the cracks is smaller than the maximum diameter of the domain that can be completely bonded. With the increase of the load and sequential cracking, debonding is triggered in correspondence to subdomains where this threshold is passed. Debonding is hence energetically favorable and releases energy continuously with the increasing load, no other transverse cracks will appear and the energy is released through debonding. In this sense, the experiments presented here are weakly coupled, for multiple fissuration and debonding do not occur simultaneously in order to release the stored elastic energy but the latter follows the former, the transition between the two phenomena being determined by the size effect. Analytic proofs of this qualitative argument are given in one dimension in León Baldelli et al. (2013).

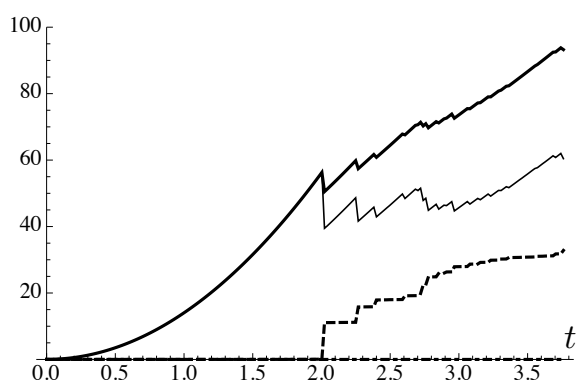

(a) In the multifissuration experiment a network of six hexagonal cracks appears at $t=$ 2.0 .

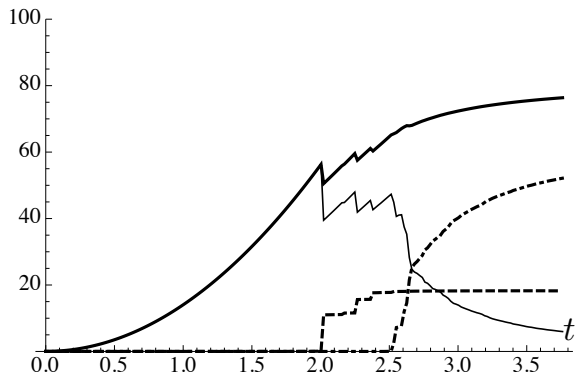

(b) In the coupled multifissuration and debonding experiment, after appearence of the network of cracks, debonding appears at $t=$ 2.50 .

Figure 10: Charts of the non-dimensional energy for the wafer experiments. Total, elastic, and surface energies are plotted with thick solid, thin solid, and dashed lines, respectively. The evolution of the initial stages of cracking is the same for both experiments. After the onset of debonding in (b), no other cracks form.

\subsection{Vinyl lettering on a metal substrate}

The last numeric experiment is inspired by a real-life example, given by the stickers identifying research labs at the Ecole Polytechnique in Palaiseau, France. A thin vinyl sticker is bonded to a metal panel and exposed to atmospheric conditions. Among others, the incident radiation from the Sun generates inelastic mismatch strains leading to transverse cracking and possibly debonding. The inelastic stresses are due to 


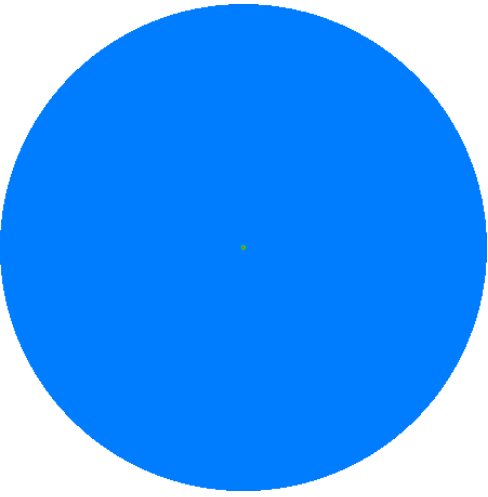

(a) $t=0.62$

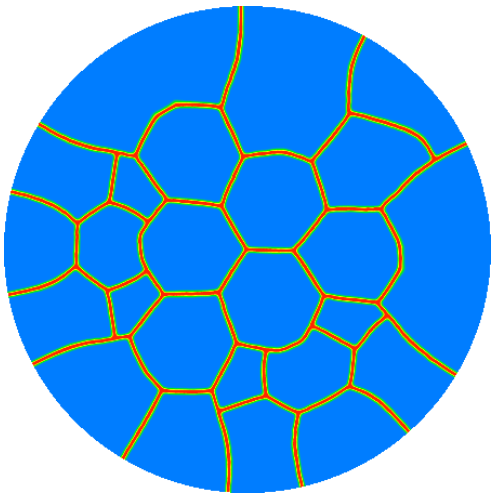

(d) $t=2.26$

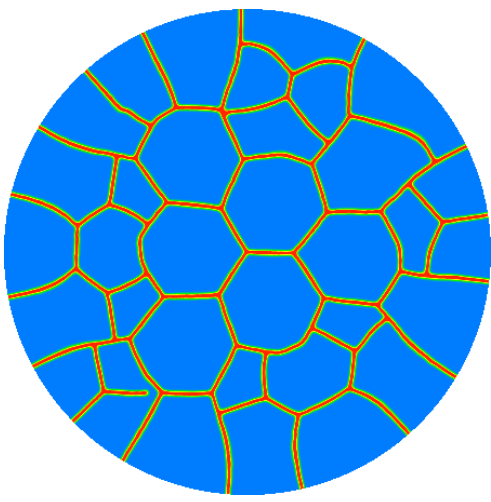

(g) $t=2.92$

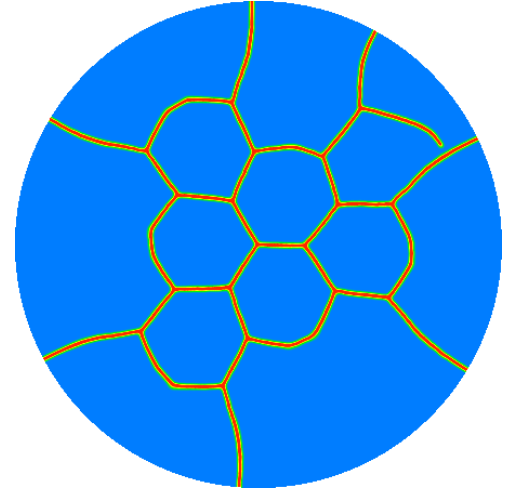

(b) $t=1.99$

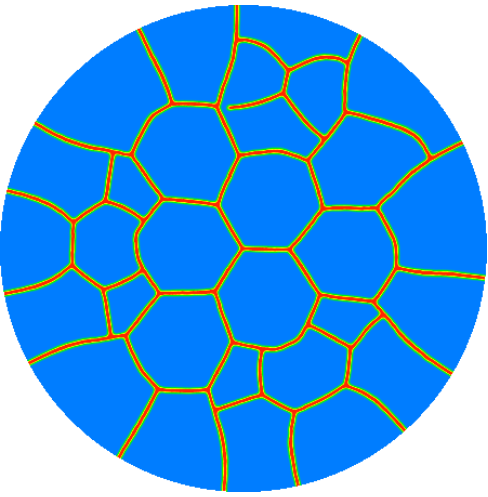

(e) $t=2.39$

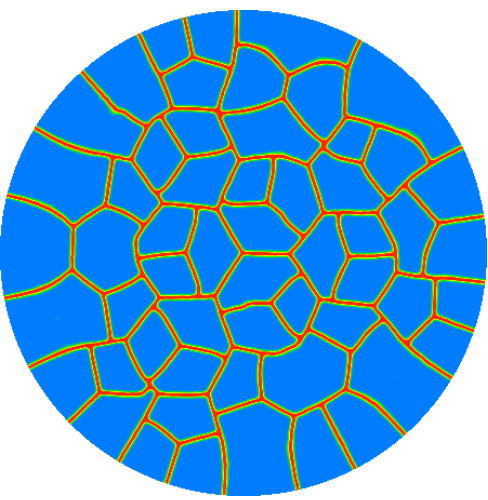

(h) $t=3.57$

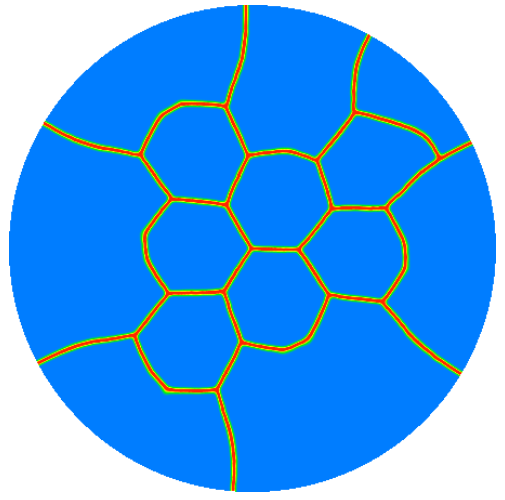

(c) $t=2.03$

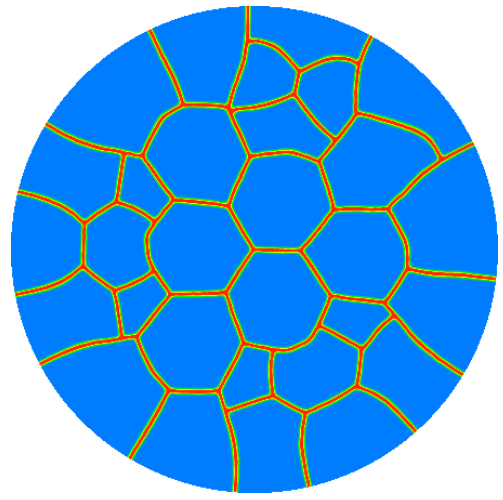

(f) $t=2.48$

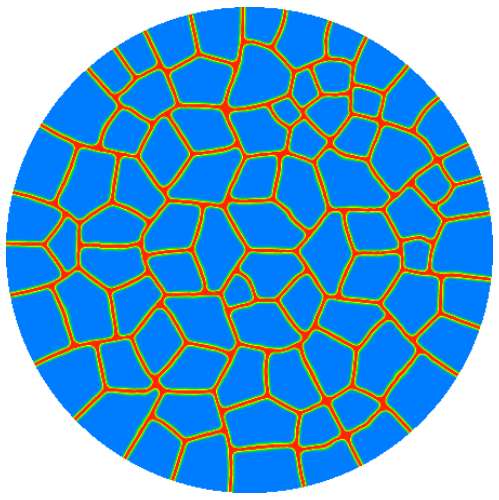

(i) $t=T_{\max }=3.76$

Figure 11: Snapshots of the fracture field in the wafer experiment under uniform load. The first fracture pattern consists of periodic hexagonal cells while for higher loadings, new cracks intersect preexisting boundaries at a right angle. 


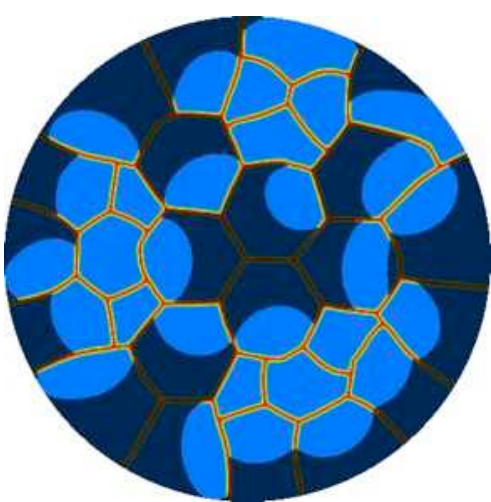

(a) $t=2.92$

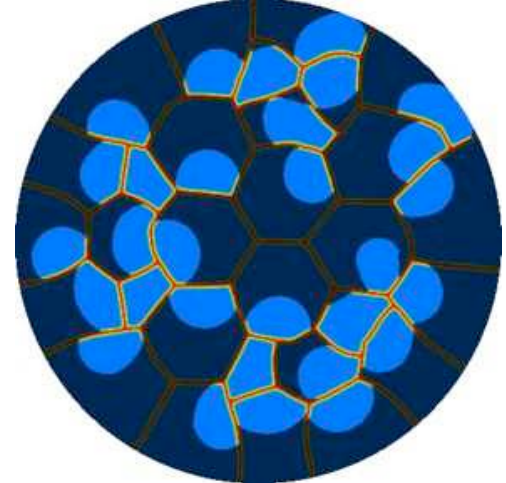

(b) $t=3.57$

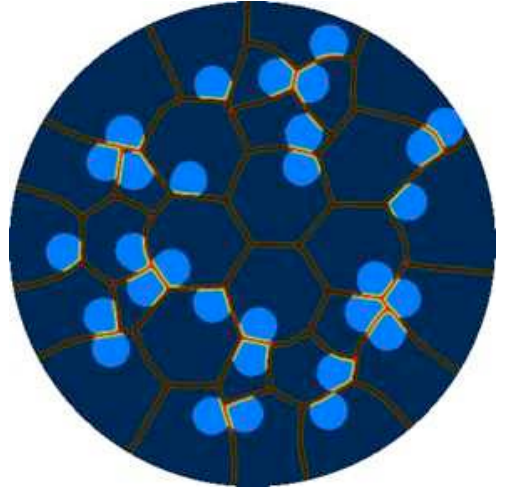

(c) $t=T_{\max }=3.76$

Figure 12: Combined fracture and debonding of a wafer. Dark areas identify debonded regions, whose first onset is at the boundaries of the largest cells. At the last time step all cells have undergone debonding.
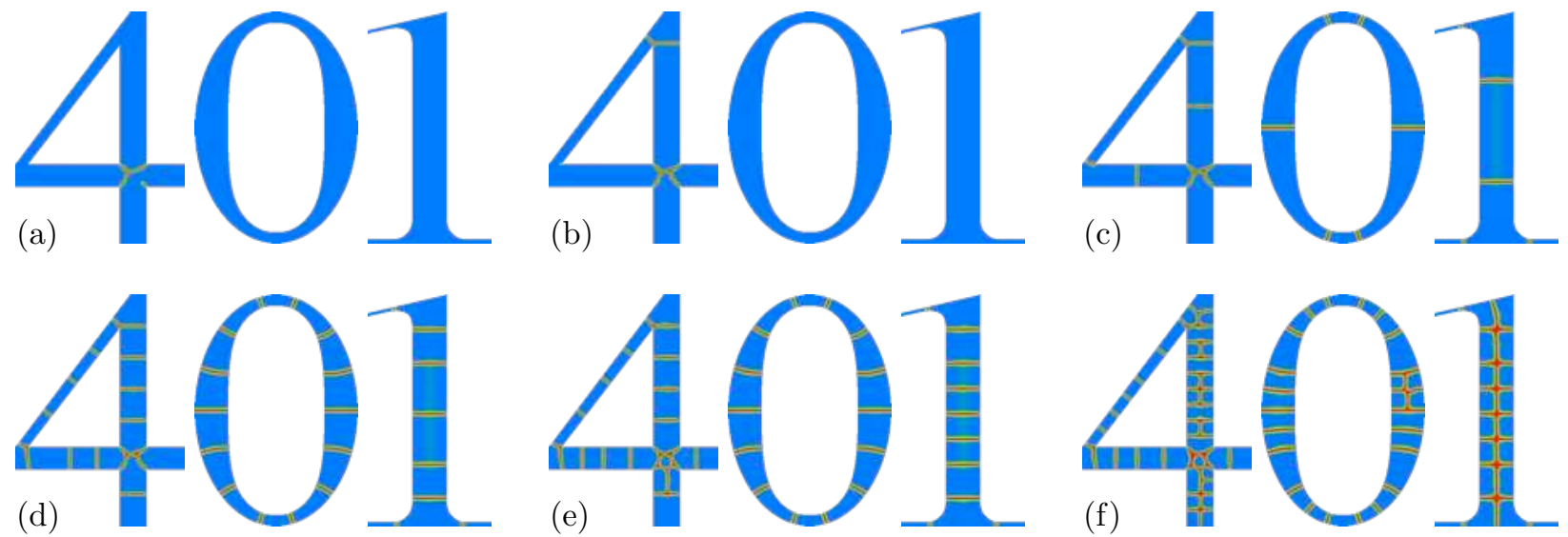

Figure 13: Computed crack pattern at (a) $t=1.05$, (b) $t=1.25$, (c) $t=1.65$, (d) $t=1.88$, (e) $t=2.15$, (f) $t=2.48$
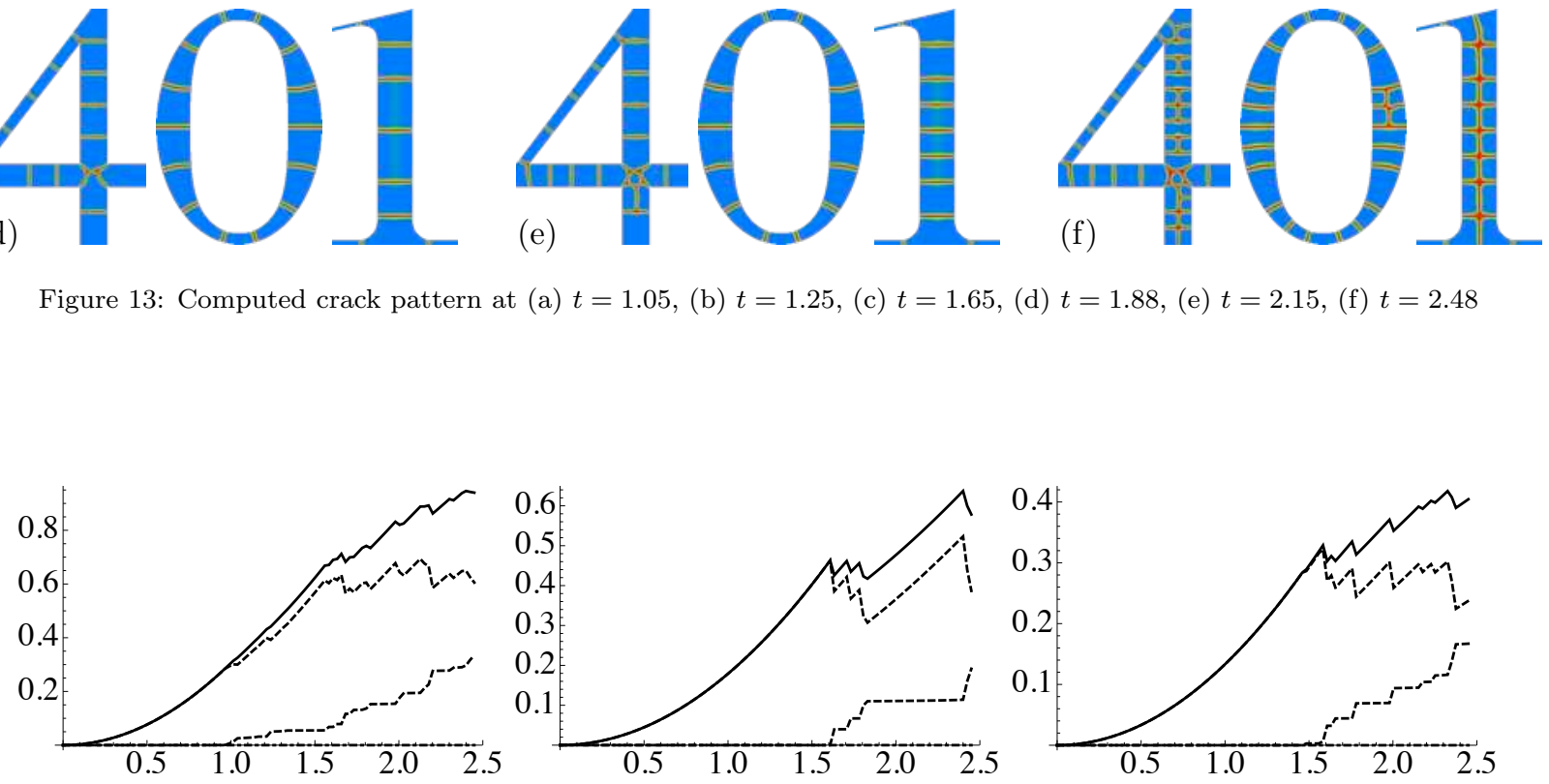

(f)

Figure 14: Energy charts for the subdomains corresponding to the digits "4", "0", and "1". 

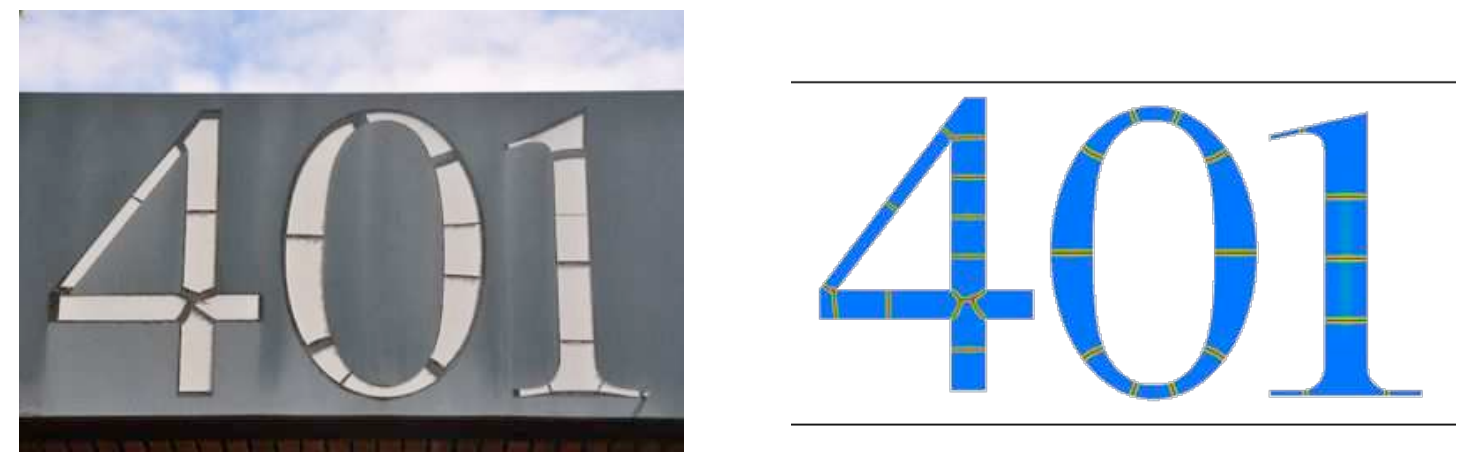

Figure 15: Cracked lettering at École Polytechnique, Palaiseau, France and numerical simulation for $t=1.75$.

shrinkage of the sticker, as it can be argued by the trace of glue left on the panel. A few panels relative to numbers in the range "401"-" 408 ", all of the same material and subject to similar loading conditions, show recurring crack patterns. One picture is reproduced in Figure 15. From the analysis of the current state, we infer qualitative informations about the evolution of the cracking process. The crack opening can be related to the evolution of the cracks: wider openings indicate cracking at earlier stages of loading. Peripheral debonding is present but limited to a tiny region, we decide for simplicity not to include its effects in the experiment. Comparing the cracks of all stickers (not shown here), the robustness of the cracks is striking. The first cracks are the ones at the center of the number "4", nucleating from the weak geometric singularities. Within the number " 0 ", the first four cracks appear in the lower and upper lobes. After, cracks open in the two longest vertical parts. The stem of the number "1" exhibits almost equidistributed cracks, the extreme aspect ratio producing almost one-dimensional solutions. Foot's and head's serifs are responsible of stress concentration and favor the appearance of cracks whereas slender segments show almost equidistributed cracks. We perform a numerical experiment meshing a domain corresponding to the number "401". Material parameters as well as numerical parameters are the same for all digits, and in order to fix debonding we set $\gamma=2 \cdot 10^{4}$. Consequently the only parameters identifying the experiment are the relative stiffness $\kappa=17.68$ (the corresponding elastic length $\ell_{e}=0.056$ ), the Poisson ratio $\nu=0.3$ and the internal length $\eta=72 \cdot 10^{-4}$. The characteristic diameter of the triangular elements is $h=12 \cdot 10^{-4}$ and the ratio $h / \eta$ is 6 . The digits " 4 ", " 0 ", " 1 " consist of $(1.6,0.6,0.3) \cdot 10^{6}$ dofs respectively. We do not model the circadian loading and impose a uniform inelastic strain increasing linearly with time. Figure 14 shows the energy evolution and Figure 13 successive snapshots of the crack field on the reference configuration. The first cracks appear at the intersection of the stem and the crossbar of the number " 4 " nucleating at the weak geometric singularities, see Figure 13(a). A triple junction is first created by three cracks originating at the North-East, North-West and South-West corners. Subsequently a third crack nucleating at the South-East corner intersects the free edge just created at a right angle. The same pattern is observed for the vinyl sticker. In Figure 13(c) the stem and crossbar of the number "4" are cracked at the center and respectively two and one cracks are produced at their intersection with the diagonal segment. In the numeric experiment, six cracks appear simultaneously in the number "0", two at each lobe (upper and lower) and a horizontal crack at the center of each of the two side arches. The crack pattern is symmetric. Here, the aspect ratio of the domain plays an important role and as the width-to-height ratio increases the cracks on the side arches are favored over those on the lobes. For the same load intensity, the number "1" shows three cracks at the serifs and two in the stem. The cracks at the serifs are favored by the sudden thickness variation and match the observed pattern. At higher loadings the periodicity of the cracks becomes prominent (Figure 13(d)) and secondary cracks (orthogonal to the former) appear (Figures 13(e-f)). A comparison of the observed patterns and our numerical experiments is shown in Figure 15. 


\section{Conclusions}

We studied the problem of fracture and debonding of a thin film bonded on an infinitely stiff substrate in the framework of the variational approach to fracture. The variational setting allowed us to perform dimensional reduction through a $\Gamma$-convergence technique and tackle the problem from the numerical point of view using a regularized formulation. The dimension reduction result accounts for the presence of the cracks and gives a limit fracture mechanics model in the case of a thin film bonded to the substrate through a soft brittle bonding layer whose fracture toughness is much smaller than the one of the film. The reduced model obtained under these scaling hypotheses may be mechanically interpreted as a brittle elastic membrane on a brittle elastic foundation. The elastic foundation term accounts for the strain energy of the bonding layer and the final model is similar to the one proposed by Xia and Hutchinson (2000) in a different setting. Fractures may appear both in the membrane and in the bonding layer. The asymptotic analysis characterizes their respective nature. In the limit two-dimensional model, transverse cracks in the membrane are curves where the displacement may jump, whilst debonding cracks are two-dimensional surfaces not associated to displacement discontinuities. The debonding condition reduces to a simple threshold criterion on the norm of the displacement. This naturally demands a different numerical treatment of the two cracking modes: debonding cracks are represented by their characteristic function, while we adopted a regularized representation of transverse cracks. We reported several numerical examples comparing our numerical approach with analytical solutions and real-life examples.

The model presented here is based on several strong hypotheses, which may be difficult to reproduce in experimental conditions. Perhaps the strongest among them is to assume a perfectly linear-elastic/brittle behavior of the bonding layer, while in real materials plastic effects may play an important role. Further work should be conducted to achieve a quantitive comparison with experimental data. From the mathematical point of view, the present dimensional reduction results should be extended to fully justify the limit model in the framework of vectorial elasticity and to consider more general scaling hypotheses on the material and geometric properties of the three-dimensional system.

Acknowledgments. The authors wish to heartily acknowledge Jean-Jacques Marigo for his guidance, unconditional support, and inspiration throughout this work.

This work has been carried on in the framework of bilateral exchange program between $\mathrm{CM}$ and $\mathrm{BB}$ financed by French National Research Council (CNRS/PICS) and a joint project EMERGENCES-UPMC between CM and JFB financed by the University Pierre et Marie Curie. DH's research was supported by the FONDECYT Iniciación Project 11110011 of the Ministry of Education of Chile. JFB was supported by the Agence Nationale de la Recherche under Grant No. ANR 10-JCJC 0106. BB's work was supported in parts by the National Science Foundation under Grant No. DMS- 0909267. CM gratefully acknowledges the funding of the ANR program T-Shock OTP J11R087.

\section{Appendix A. Special functions of bounded variation}

The object of this section is to recall some notations about the space of special functions of bounded variation. Let $U$ be a bounded open subset of $\mathbb{R}^{n}$. The Lebesgue measure is denoted by $\mathcal{L}^{n}$ while $\mathcal{H}^{k}$ stands for the $k$-dimensional Hausdorff measure.

The space $S B V(U)$ of special functions of bounded variation is made of all (scalar) functions $u \in L^{1}(U)$ such that the distributional derivative $D u$ is a bounded Radon measure which can be written as

$$
D u=\nabla u \mathcal{L}^{n}+\left(u^{+}-u^{-}\right) \nu_{u} \mathcal{H}^{n-1}\left\llcorner J_{u} .\right.
$$

In the previous formula:

- $\nabla u \in L^{1}\left(U ; \mathbb{R}^{n}\right)$ denotes the approximate gradient, i.e. the density of $D u$ with respect to the Lebesgue measure $\mathcal{L}^{n}$;

- $J_{u}$ is the jump set of $u$. It is a rectifiable set whose unit normal is denoted $\nu_{u}$; 
- $u^{ \pm}$are the one-sided traces of $u$ on $J_{u}$.

We refer to Ambrosio et al. (2000) for a detailed description of this space.

\section{Appendix B. Proof of the dimensional reduction for scalar elasticity}

This section is devoted to give a rigorous proof of Theorem 3.1. As explained in Section 3.2, it will be obtained in three steps: we first show that sequences with uniformly bounded energy admit converging subsequences. Then we prove a lower bound for the limit energy. Eventually, we show that this lower bound is optimal through the construction of a recovery sequence which gives an upper bound.

Let us recall that the three-dimensional energy is defined as in (12) by

$$
\begin{aligned}
\mathcal{E}_{\varepsilon}(u)= & \frac{\mu_{f}}{2}\left(\int_{\Omega_{f}}\left(\left|\nabla^{\prime} u-\epsilon_{0}^{\prime}\right|^{2}+\frac{1}{\varepsilon^{2}}\left(\partial_{3} u\right)^{2}\right) \mathrm{d} x+\rho_{\mu} \int_{\Omega_{b}}\left(\varepsilon^{2}\left|\nabla^{\prime} u-\epsilon_{0}^{\prime}\right|^{2}+\left(\partial_{3} u\right)^{2}\right) \mathrm{d} x\right) \\
& +G_{f}\left(\int_{J_{u} \cap \Omega_{f}}\left|\left(\nu_{u}^{\prime}, \frac{1}{\varepsilon}\left(\nu_{u}\right)_{3}\right)\right| \mathrm{d} \mathcal{H}^{2}+\rho_{G} \int_{J_{u} \cap \Omega_{b}}\left|\left(\varepsilon \nu_{u}^{\prime},\left(\nu_{u}\right)_{3}\right)\right| \mathrm{d} \mathcal{H}^{2}\right)
\end{aligned}
$$

for any $u$ is the space of all kinematically admissible displacements

$$
\mathcal{C}_{w}(\Omega):=\left\{u \in S B V(\Omega): u=w \text { a.e. in } \Omega_{s}, \text { and }\|u\|_{L^{\infty}\left(\Omega_{f}\right)} \leq M\right\} .
$$

We henceforth assume that $\epsilon_{0}^{\prime} \in L^{2}\left(\omega ; \mathbb{R}^{2}\right)$ and $w \in H^{1}(\omega) \cap L^{\infty}(\omega)$.

Appendix B.1. Compactness in the film

Proposition Appendix B.1. Let $\left(\varepsilon_{n}\right) \searrow 0^{+}$and $\left(u_{n}\right) \subset \mathcal{C}_{w}(\Omega)$ be sequences satisfying

$$
\sup _{n \in \mathbb{N}} \mathcal{E}_{\varepsilon_{n}}\left(u_{n}\right)<\infty .
$$

Then there exist a subsequence (not relabeled) and $u \in S B V\left(\Omega_{f}\right)$ such that

$$
\left\{\begin{array}{l}
u_{n} \rightarrow u \text { strongly in } L^{2}\left(\Omega_{f}\right), \\
u_{n} \rightarrow u \text { weakly* in } L^{\infty}\left(\Omega_{f}\right), \\
\nabla u_{n} \rightarrow \nabla u \text { weakly in } L^{2}\left(\Omega_{f} ; \mathbb{R}^{3}\right) .
\end{array}\right.
$$

Moreover, $\partial_{3} u=0$ a.e. in $\Omega_{f}$, and $\left(\nu_{u}\right)_{3}=0 \mathcal{H}^{2}$-a.e. on $J_{u} \cap \Omega_{f}$. Hence, the limit displacement field $u$ (can be identified to a function that) belongs to $S B V(\omega)$, it satisfies $\|u\|_{L^{\infty}(\omega)} \leq M$, and

$$
\left\{\begin{array}{l}
L \int_{\omega}\left|\nabla^{\prime} u-\epsilon_{0}^{\prime}\right|^{2} d x^{\prime} \leq \liminf _{n \rightarrow \infty} \int_{\Omega_{f}}\left(\left|\nabla^{\prime} u_{n}-\epsilon_{0}^{\prime}\right|^{2}+\frac{1}{\varepsilon_{n}^{2}}\left|\partial_{3} u_{n}\right|^{2}\right) d x \\
L \mathcal{H}^{1}\left(J_{u}^{\prime}\right) \leq \liminf _{n \rightarrow \infty} \int_{\Omega_{f} \cap J_{u_{n}}}\left|\left(\left(\nu_{u_{n}}\right)^{\prime}, \frac{1}{\varepsilon_{n}}\left(\nu_{u_{n}}\right)_{3}\right)\right| d \mathcal{H}^{2} .
\end{array}\right.
$$

Proof. According to (B.1), the definition of the energy $\mathcal{E}_{\varepsilon_{n}}$ and of that of the space of kinematically admissible displacements $\mathcal{C}_{w}(\Omega)$, we have the following bounds

$$
\left\|u_{n}\right\|_{L^{\infty}\left(\Omega_{f}\right)}+\left\|\nabla u_{n}\right\|_{L^{2}\left(\Omega_{f} ; \mathbb{R}^{3}\right)}+\mathcal{H}^{2}\left(J_{u_{n}} \cap \Omega_{f}\right) \leq C
$$

for some constant $C>0$ independent of $n$. According to Ambrosio's compactness Theorem in $S B V$ (see (Ambrosio et al., 2000, Thms 4.7 and 4.8)), we deduce the existence of a subsequence $\left(u_{n_{k}}\right) \subset\left(u_{n}\right)$ and a function $u \in S B V\left(\Omega_{f}\right)$ such that $u_{n_{k}} \rightarrow u$ strongly in $L^{2}\left(\Omega_{f}\right), u_{n_{k}} \rightarrow u$ weakly* in $L^{\infty}\left(\Omega_{f}\right), \nabla u_{n_{k}} \rightarrow \nabla u$ weakly in $L^{2}\left(\Omega_{f} ; \mathbb{R}^{3}\right)$, and

$$
\mathcal{H}^{2}\left(J_{u} \cap \Omega_{f}\right) \leq \liminf _{k \rightarrow+\infty} \mathcal{H}^{2}\left(J_{u_{n_{k}}} \cap \Omega_{f}\right) .
$$


Let us prove that $u$ is actually independent of $x_{3}$. Using the expression of the energy in the film, we deduce that

$$
\int_{\Omega_{f}}\left|\partial_{3} u_{n_{k}}\right|^{2} d x+\int_{\Omega_{f} \cap J_{u_{n_{k}}}}\left|\left(\nu_{u_{n_{k}}}\right)_{3}\right| d \mathcal{H}^{2} \leq C \varepsilon_{n_{k}} \rightarrow 0 .
$$

Since the left hand side of the previous inequality is lower semicontinuous with respect to the convergences established for $\left(u_{n_{k}}\right)$ (see e.g. Babadjian (2006); Braides and Fonseca (2001); Bouchitte et al. (2002)), we conclude that $\partial_{3} u=0$ a.e. in $\Omega_{f}$, and $\left(\nu_{u}\right)_{3}=0 \mathcal{H}^{2}$-a.e. on $J_{u} \cap \Omega_{f}$. This implies that the distributional derivative $D_{3} u=0$ in $\mathcal{D}^{\prime}\left(\Omega_{f}\right)$, and thus the limit displacement field $u$ (can be identified to a function that) belongs to $S B V(\omega)$.

By definition of $\mathcal{C}_{w}(\Omega)$, we have that $\left\|u_{n}\right\|_{L^{\infty}\left(\Omega_{f}\right)} \leq M$. Therefore, we deduce by lower semicontinuity of the norm with respect to weak* convergence in $L^{\infty}\left(\Omega_{f}\right)$ that $\|u\|_{L^{\infty}(\omega)} \leq M$.

Since $u$ is independent of $x_{3}$, the approximate gradient is given by $\nabla u=\left(\nabla^{\prime} u, 0\right)$ and the jump set can be written as $J_{u}=J_{u}^{\prime} \times(0, L)$ for some 1-rectifiable set $J_{u}^{\prime} \subset \omega$. Finally, since $\varepsilon_{n_{k}} \leq 1$, we infer that

$$
L \int_{\omega}\left|\nabla^{\prime} u-\epsilon_{0}^{\prime}\right|^{2} d x^{\prime} \leq \liminf _{k \rightarrow \infty} \int_{\Omega_{f}}\left|\nabla u_{n_{k}}-\left(\epsilon_{0}^{\prime}, 0\right)\right|^{2} d x \leq \liminf _{k \rightarrow \infty} \int_{\Omega_{f}}\left(\left|\nabla^{\prime} u_{n_{k}}-\epsilon_{0}^{\prime}\right|^{2}+\frac{1}{\varepsilon_{n_{k}}^{2}}\left|\partial_{3} u_{n_{k}}\right|^{2}\right) d x
$$

and

$$
L \mathcal{H}^{1}\left(J_{u}^{\prime}\right)=\mathcal{H}^{2}\left(J_{u} \cap \Omega_{f}\right) \leq \liminf _{k \rightarrow \infty} \mathcal{H}^{2}\left(J_{u_{n_{k}}} \cap \Omega_{f}\right) \leq \liminf _{k \rightarrow \infty} \int_{\Omega_{f} \cap J_{u_{n_{k}}}}\left|\left(\left(\nu_{u_{n_{k}}}\right)^{\prime}, \frac{1}{\varepsilon_{n_{k}}}\left(\nu_{u_{n_{k}}}\right)_{3}\right)\right| d \mathcal{H}^{2},
$$

which completes the proof of the proposition.

The previous compactness result suggests to define the set

$$
\mathcal{C}(\omega)=\left\{u \in S B V(\omega):\|u\|_{L^{\infty}(\omega)} \leq M\right\} .
$$

For any $u \in \mathcal{C}(\omega)$, we recall that the reduced two-dimensional energy is defined by

$$
\mathcal{E}_{0}(u):=\frac{L \mu_{f}}{2} \int_{\omega}\left|\nabla^{\prime} u-\epsilon_{0}^{\prime}\right|^{2} d x^{\prime}+\frac{L \mu_{b}}{2 h_{f} h_{b}} \int_{\omega \backslash \Delta_{u}}|u-w|^{2} d x^{\prime}+L G_{f} \mathcal{H}^{1}\left(J_{u}^{\prime}\right)+\frac{L G_{b}}{h_{f}} \mathcal{H}^{2}\left(\Delta_{u}\right),
$$

where

$$
\Delta_{u}:=\left\{x^{\prime} \in \omega:\left|u\left(x^{\prime}\right)-w\left(x^{\prime}\right)\right|>u_{d}:=\sqrt{\frac{2 G_{b} h_{b}}{\mu_{b}}}\right\}
$$

is the delamination set.

\section{Appendix B.2. Lower bound}

Proposition Appendix B.2. For any $u \in \mathcal{C}(\omega)$, and any sequences $\left(\varepsilon_{n}\right) \searrow 0^{+}$and $\left(u_{n}\right) \subset \mathcal{C}_{w}(\Omega)$ such that $u_{n} \rightarrow u$ strongly in $L^{2}\left(\Omega_{f}\right)$, then

$$
\mathcal{E}_{0}(u) \leq \liminf _{n \rightarrow \infty} \mathcal{E}_{\varepsilon_{n}}\left(u_{n}\right)
$$

Proof. Let us extract a subsequence (not relabeled) such that the previous lim inf is actually a limit. Then for $n$ large enough, one has

$$
\mathcal{E}_{\varepsilon_{n}}\left(u_{n}\right) \leq C
$$

for some constant $C>0$. According to Proposition Appendix B.1, $u_{n} \rightarrow u$ strongly in $L^{2}\left(\Omega_{f}\right), u_{n} \rightarrow u$ weakly* in $L^{\infty}\left(\Omega_{f}\right), \nabla u_{n} \rightarrow \nabla u$ weakly in $L^{2}\left(\Omega_{f} ; \mathbb{R}^{3}\right)$, and

$$
\left\{\begin{array}{l}
\mu_{f} L \int_{\omega}\left|\nabla^{\prime} u-\epsilon_{0}^{\prime}\right|^{2} d x^{\prime} \leq \liminf _{n \rightarrow \infty} \mu_{f} \int_{\Omega_{f}}\left(\left|\nabla^{\prime} u_{n}-\epsilon_{0}^{\prime}\right|^{2}+\frac{1}{\varepsilon_{n}^{2}}\left|\partial_{3} u_{n}\right|^{2}\right) d x, \\
G_{f} L \mathcal{H}^{1}\left(J_{u}^{\prime}\right) \leq \liminf _{n \rightarrow \infty} G_{f} \int_{\Omega_{f} \cap J_{u_{n}}}\left|\left(\left(\nu_{u_{n}}\right)^{\prime}, \frac{1}{\varepsilon_{n}}\left(\nu_{u_{n}}\right)_{3}\right)\right| d \mathcal{H}^{2} .
\end{array}\right.
$$


Consequently, it is enough to consider the energy in the bonding layer $\Omega_{b}$, and to check that

$$
\begin{aligned}
& \frac{L \mu_{b}}{h_{f} h_{b}} \int_{\omega \backslash \Delta_{u}}|u-w|^{2} d x^{\prime}+\frac{L G_{b}}{h_{f}} \mathrm{~d} \mathcal{H}^{2}\left(\Delta_{u}\right) \\
& \quad \leq \liminf _{n \rightarrow \infty}\left(\mu_{f} \rho_{\mu} \int_{\Omega_{b}}\left(\varepsilon_{n}^{2}\left|\nabla^{\prime} u_{n}\right|^{2}+\left|\partial_{3} u_{n}\right|^{2}\right) d x+G_{f} \rho_{G} \int_{\Omega_{b} \cap J_{u_{n}}}\left|\left(\varepsilon_{n}\left(\nu_{u_{n}}\right)^{\prime},\left(\nu_{u_{n}}\right)_{3}\right)\right| \mathrm{d} \mathcal{H}^{2}\right)
\end{aligned}
$$

The rest of the proof is devoted to show (B.4). The main difficulty consists in defining the debonding set. This is performed as follows: let $x^{\prime} \in \omega$, we define the transverse section of the jump set of $u_{n}$ by $J_{n}^{x^{\prime}}:=\left\{x_{3} \in\left(-2 L \rho_{h}, L\right):\left(x^{\prime}, x_{3}\right) \in J_{u_{n}}\right\}$ and

$$
\Delta_{n}:=\left\{x^{\prime} \in \omega: J_{n}^{x^{\prime}} \neq \emptyset\right\}
$$

The set $\Delta_{n}$ is made of all points in the plane from which the vertical section intersects the jump set $J_{u_{n}}$ or, in other words, $\Delta_{n}$ is the orthogonal projection of $J_{u_{n}}$ on $\omega$. It can be interpreted as an approximation of the debonding zone. Unfortunately, it is not clear how to show that it converges to some (debonding) set because we only control the $L^{\infty}(\omega)$ norm of its characteristic function. Therefore, possibly for a subsequence (not relabeled), one can find some $\vartheta \in L^{\infty}(\omega ;[0,1])$ such that $\chi_{\Delta_{n}} \rightarrow \vartheta$ weakly* in $L^{\infty}(\omega ;[0,1])$.

Step 1. We first obtain that, outside the debonding set $\Delta_{n}$, the trace $u_{n}(\cdot, 0)$ of $u_{n}$ at the interface $\omega \times\{0\}$ between the film and the bonding layer converges strongly in $L^{2}(\omega)$ to the limit displacement $u$ (which is independent of $x_{3}$ ). In absence of debonding, this property is standard as a consequence of the compactness of the trace operator from $H^{1}\left(\Omega_{f}\right)$ to $L^{2}(\omega)$. However, in the presence of debonding, since $u_{n}$ is a $S B V\left(\Omega_{f}\right)$ function, this property does not hold anymore. What makes the argument work in our case is that the function $x_{3} \mapsto u_{n}\left(x^{\prime}, x_{3}\right)$ is Sobolev whenever $x^{\prime}$ lives outside the debonding set $\Delta_{n}$. To be more precise, let us show that

$$
\int_{\omega \backslash \Delta_{n}}\left|u\left(x^{\prime}\right)-u_{n}\left(x^{\prime}, 0\right)\right|^{2} d x^{\prime} \rightarrow 0 .
$$

For each $x^{\prime} \in \omega$, let us define $u_{n}^{x^{\prime}}\left(x_{3}\right):=u_{n}\left(x^{\prime}, x_{3}\right)$. According to slicing properties of $S B V$ functions (see (Ambrosio et al., 2000, Thms 3.107 and 3.108)), we have $u_{n}^{x^{\prime}} \in S B V\left(-2 L \rho_{h}, L\right)$, and $J_{u_{n}^{x^{\prime}}}=J_{n}^{x^{\prime}}$ for a.e. $x^{\prime} \in \omega$. Hence by definition of $\Delta_{n}$, we deduce that $u_{n}^{x^{\prime}} \in H^{1}\left(-2 L \rho_{h}, L\right)$ for a.e. $x^{\prime} \in \omega \backslash \Delta_{n}$. In addition, for a.e. $x_{3} \in(0, L)$, we have $\left(u_{n}^{x^{\prime}}\right)^{\prime}\left(x_{3}\right)=\partial_{3} u_{n}\left(x^{\prime}, x_{3}\right)$ (by (Ambrosio et al., 2000, Prop. 4.35)), and

$$
\left|u_{n}\left(x^{\prime}, x_{3}\right)-u_{n}\left(x^{\prime}, 0\right)\right|=\left|u_{n}^{x^{\prime}}\left(x_{3}\right)-u_{n}\left(x^{\prime}, 0\right)\right| \leq \int_{0}^{x_{3}}\left|\left(u_{n}^{x^{\prime}}\right)^{\prime}(s)\right| d s \leq \int_{0}^{L}\left|\partial_{3} u_{n}\left(x^{\prime}, s\right)\right| d s .
$$

Integrating with respect to $x_{3} \in(0, L)$ and $x^{\prime} \in \omega \backslash \Delta_{n}$, the Cauchy-Schwarz inequality and (B.2) yield

$$
\begin{array}{rl}
\int_{\omega \backslash \Delta_{n}} \int_{0}^{L}\left|u_{n}\left(x^{\prime}, x_{3}\right)-u_{n}\left(x^{\prime}, 0\right)\right|^{2} & d x_{3} d x^{\prime} \\
\leq L^{2} \int_{\omega \backslash \Delta_{n}} \int_{0}^{L}\left|\partial_{3} u_{n}\left(x^{\prime}, x_{3}\right)\right|^{2} d x_{3} d x^{\prime} \leq L^{2} \int_{\Omega_{f}}\left|\partial_{3} u_{n}(x)\right|^{2} d x \leq C \varepsilon_{n}^{2} .
\end{array}
$$

In addition, since $u_{n} \rightarrow u$ strongly in $L^{2}\left(\Omega_{f}\right)$, and $u$ is independent of $x_{3}$, we finally obtain (B.5).

Step 2. We next show lower bounds in terms of the density $\vartheta$ of debonding for the volume and surface energies in the bonding layer:

$$
\frac{L \mu_{b}}{h_{f} h_{b}} \int_{\omega}(1-\vartheta)(u-w)^{2} d x^{\prime} \leq \liminf _{n \rightarrow \infty} \mu_{f} \rho_{\mu} \int_{\Omega_{b}}\left(\varepsilon_{n}^{2}\left|\nabla^{\prime} u_{n}-\epsilon_{0}^{\prime}\right|^{2}+\left|\partial_{3} u_{n}\right|^{2}\right) d x
$$


and

$$
\frac{L G_{b}}{h_{f}} \int_{\omega} \vartheta d x^{\prime} \leq \liminf _{n \rightarrow \infty} G_{f} \rho_{G} \int_{\Omega_{b} \cap J_{u_{n}}}\left|\left(\varepsilon_{n}\left(\nu_{u_{n}}\right)^{\prime},\left(\nu_{u_{n}}\right)_{3}\right)\right| d \mathcal{H}^{2} .
$$

Intuitively the term of order $\varepsilon_{n}^{2}$ in (B.6) and the term of order $\varepsilon_{n}$ in (B.7) can be neglected so that we only focus on terms of order 1. Let us start by proving (B.6). Using the Cauchy-Schwarz inequality and the fact that $\chi_{\Delta_{n}} \rightarrow \vartheta$ weakly* in $L^{\infty}(\omega ;[0,1])$, we infer that

$$
\begin{aligned}
\liminf _{n \rightarrow \infty} \int_{\Omega_{b}} & \left(\varepsilon_{n}^{2}\left|\nabla^{\prime} u_{n}-\epsilon_{0}^{\prime}\right|^{2}+\left|\partial_{3} u_{n}\right|^{2}\right) d x \\
& \geq \liminf _{n \rightarrow \infty} \int_{\omega \backslash \Delta_{n}} \int_{-L \rho_{h}}^{0}\left|\partial_{3} u_{n}\right|^{2} d x_{3} d x^{\prime} \geq \liminf _{n \rightarrow \infty} \frac{1}{L \rho_{h}} \int_{\omega \backslash \Delta_{n}}\left(\int_{-L \rho_{h}}^{0} \partial_{3} u_{n} d x_{3}\right)^{2} d x^{\prime} \\
& \geq \frac{1}{L \rho_{h}} \int_{\omega}(1-\vartheta)(u-w)^{2} d x^{\prime}+\liminf _{n \rightarrow \infty} \frac{1}{L \rho_{h}} \int_{\omega \backslash \Delta_{n}}\left[\left(\int_{-L \rho_{h}}^{0} \partial_{3} u_{n} d x_{3}\right)^{2}-(u-w)^{2}\right] d x^{\prime} .
\end{aligned}
$$

Since $u_{n}\left(x^{\prime}, \cdot\right) \in H^{1}\left(-2 L \rho_{h}, L\right)$ for a.e. $x \in \omega \backslash \Delta_{n}$, then the trace $u_{n}\left(\cdot,-2 L \rho_{h}\right)$ of $u_{n}$ at the interface $\left\{x_{3}=-2 L \rho_{h}\right\}$ between the bonding layer and the substrate satisfies $u_{n}\left(x^{\prime},-2 L \rho_{h}\right)=w\left(x^{\prime}\right)$, and thus

$$
\int_{\omega \backslash \Delta_{n}}\left[\left(\int_{-L \rho_{h}}^{0} \partial_{3} u_{n} d x_{3}\right)^{2}-(u-w)^{2}\right] d x^{\prime}=\int_{\omega \backslash \Delta_{n}}\left[\left(u_{n}\left(x^{\prime}, 0\right)-w\left(x^{\prime}\right)\right)^{2}-\left(u\left(x^{\prime}\right)-w\left(x^{\prime}\right)\right)^{2}\right] d x^{\prime} .
$$

Using now (B.5), the fact that $u_{n}$ (and hence its trace $u_{n}(\cdot, 0)$ ) is uniformly bounded by $M$, and the Cauchy-Schwarz inequality we deduce that

$$
\int_{\omega \backslash \Delta_{n}}\left[\left(u_{n}\left(x^{\prime}, 0\right)-w\left(x^{\prime}\right)\right)^{2}-\left(u\left(x^{\prime}\right)-w\left(x^{\prime}\right)\right)^{2}\right] d x^{\prime} \rightarrow 0 .
$$

Thus (B.6) follows from (B.8).

We next prove (B.7). Let us denote by $\pi: \mathbb{R}^{3} \rightarrow \mathbb{R}^{2} \times\{0\}$ the orthogonal projection onto the hyperplane $\left\{x_{3}=0\right\}$. Then

$$
\liminf _{n \rightarrow \infty} \int_{\Omega_{b} \cap J_{u_{n}}}\left|\left(\varepsilon_{n}\left(\nu_{u_{n}}\right)^{\prime},\left(\nu_{u_{n}}\right)_{3}\right)\right| d \mathcal{H}^{2} \geq \liminf _{n \rightarrow \infty} \int_{\Omega_{b} \cap J_{u_{n}}}\left|\left(\nu_{u_{n}}\right)_{3}\right| d \mathcal{H}^{2} .
$$

Thanks to the coarea formula (see (Ambrosio et al., 2000, Theorem 2.93)), we infer that

$$
\begin{aligned}
& \int_{\Omega_{b} \cap J_{u_{n}}}\left|\left(\nu_{u_{n}}\right)_{3}\right| d \mathcal{H}^{2}=\int_{\mathbb{R}^{2}} \mathcal{H}^{0}\left(\Omega_{b} \cap J_{u_{n}} \cap \pi^{-1}\left(x^{\prime}\right)\right) d \mathcal{H}^{2}\left(x^{\prime}\right) \\
&=\int_{\mathbb{R}^{2}} \mathcal{H}^{0}\left(J_{u_{n}} \cap \pi^{-1}\left(x^{\prime}\right)\right) d \mathcal{H}^{2}\left(x^{\prime}\right)-\int_{\mathbb{R}^{2}} \mathcal{H}^{0}\left(\Omega_{f} \cap J_{u_{n}} \cap \pi^{-1}\left(x^{\prime}\right)\right) d \mathcal{H}^{2}\left(x^{\prime}\right) .
\end{aligned}
$$

Therefore, since $\mathcal{H}^{0}\left(J_{u_{n}} \cap \pi^{-1}\left(x^{\prime}\right)\right) \geq 1$ for all $x^{\prime} \in \pi\left(J_{u_{n}}\right)=\Delta_{n}$, we obtain, using again the coarea formula in $\Omega_{f}$, that

$$
\int_{\mathbb{R}^{2}} \mathcal{H}^{0}\left(J_{u_{n}} \cap \pi^{-1}\left(x^{\prime}\right)\right) d \mathcal{H}^{2}\left(x^{\prime}\right)=\int_{\Delta_{n}} \mathcal{H}^{0}\left(J_{u_{n}} \cap \pi^{-1}\left(x^{\prime}\right)\right) d \mathcal{H}^{2}\left(x^{\prime}\right) \geq \mathcal{H}^{2}\left(\Delta_{n}\right) .
$$

On the other hand, in view of the surface energy estimate in the film (B.2), we get that

$$
\int_{\Delta_{n}} \mathcal{H}^{0}\left(\Omega_{f} \cap J_{u_{n}} \cap \pi^{-1}\left(x^{\prime}\right)\right) d \mathcal{H}^{2}\left(x^{\prime}\right)=\int_{\Omega_{f} \cap J_{u_{n}}}\left|\left(\nu_{u_{n}}\right)_{3}\right| d \mathcal{H}^{2} \leq C \varepsilon_{n} \rightarrow 0 .
$$


We thus obtain that

$$
\liminf _{n \rightarrow \infty} \int_{\Omega_{b} \cap J_{u_{n}}}\left|\left(\varepsilon_{n}\left(\nu_{u_{n}}\right)^{\prime},\left(\nu_{u_{n}}\right)_{3}\right)\right| d \mathcal{H}^{2} \geq \liminf _{n \rightarrow \infty} \mathcal{H}^{2}\left(\Delta_{n}\right)=\int_{\omega} \vartheta d x^{\prime}
$$

which completes the proof of (B.7).

Step 3. Let us prove that

$$
\frac{L \mu_{b}}{2 h_{f} h_{b}} \int_{\omega}(1-\vartheta)(u-w)^{2} d x^{\prime}+\frac{L G_{b}}{h_{f}} \int_{\omega} \vartheta d x^{\prime} \geq \frac{L \mu_{b}}{2 h_{f} h_{b}} \int_{\omega \backslash \Delta_{u}}|u-w|^{2} d x^{\prime}+\frac{L G_{b}}{h_{f}} \mathcal{H}^{2}\left(\Delta_{u}\right),
$$

where $\Delta_{u}$ is the debonding set defined by (15). Clearly, one has

$$
\frac{L \mu_{b}}{2 h_{f} h_{b}} \int_{\omega}(1-\vartheta)(u-w)^{2} d x^{\prime}+\frac{L G_{b}}{h_{f}} \int_{\omega} \vartheta d x^{\prime} \geq \frac{L}{h_{f}} \int_{\omega} \inf _{\eta \in[0,1]}\left[\eta\left(G_{b}-\frac{\mu_{b}}{2 h_{b}}(u-w)^{2}\right)+\frac{\mu_{b}}{2 h_{b}}(u-w)^{2}\right] d x^{\prime} .
$$

It is easy to check that a minimizer $\eta^{*}$ in $[0,1]$ of

$$
\eta \mapsto \eta\left(G_{b}-\frac{\mu_{b}}{2 h_{b}}(u-w)^{2}\right)+\frac{\mu_{b}}{2 h_{b}}(u-w)^{2}
$$

is given by

$$
\eta^{*}=\chi_{\left\{|u-w|>\sqrt{\frac{2 G_{b} h_{b}}{\mu_{b}}}\right\}}\left(x^{\prime}\right)
$$

and (B.9) follows from the definition (15) of the debonding set.

Appendix B.3. Upper bound and existence of a recovery sequence

Proposition Appendix B.3. For any $u \in \mathcal{C}(\omega)$ and any sequence $\left(\varepsilon_{n}\right) \searrow 0^{+}$, there exists $\left(\bar{u}_{n}\right) \subset \mathcal{C}_{w}(\Omega)$ such that $\bar{u}_{n} \rightarrow u$ strongly in $L^{2}(\Omega)$ and

$$
\mathcal{E}_{0}(u) \geq \limsup _{n \rightarrow \infty} \mathcal{E}_{\varepsilon_{n}}\left(\bar{u}_{n}\right) .
$$

Proof. In order to get an intuition of the form of the recovery sequence, let us analyze what would make optimal the lower bound established in Proposition Appendix B.2. Concerning the part in the film, we expect a displacement independent of the transverse variable $x_{3}$ in order to ensure that the term of order $1 / \varepsilon_{n}^{2}$ in the bulk energy, and that of order $1 / \varepsilon_{n}$ in the surface energy, do not blow up. Concerning the bonding layer, as already observed in the proof of the lower bound, we expect no contributions from the in-plane strain and the out-of-plane cracks since these terms vanish as the thickness tends to zero. On the other hand, according to estimate (B.8), we used the Cauchy-Schwarz inequality for the function $x_{3} \mapsto \partial_{3} u\left(x^{\prime}, x_{3}\right)$ when $x^{\prime}$ lives outside the debonding set. It is known that such inequality is an equality whenever the function is constant. Therefore, when $x^{\prime}$ does not belong to the debonding set, we expect that the function $x_{3} \mapsto u\left(x^{\prime}, x_{3}\right)$ is affine, joining continuously the prescribed displacement $w\left(x^{\prime}\right)$ on the substrate and the displacement $u\left(x^{\prime}\right)$ of the film. Finally, if $x^{\prime}$ is a debonded point, then the displacement of the film does not match that of the substrate.

Let us make this observation rigorous. By the coarea formula in $B V$ ((Ambrosio et al., 2000, Theorem $3.40)$ ), there exists a sequence $\left(t_{k}\right) \searrow u_{d}$ such that the sets $\left\{|u-w| \leq t_{k}\right\}$ have finite perimeter for each $k \in \mathbb{N}$. Let us define

$$
u^{k}\left(x^{\prime}, x_{3}\right):= \begin{cases}u\left(x^{\prime}\right) & \text { if } \quad\left(x^{\prime}, x_{3}\right) \in \Omega_{f}, \\
\left(\frac{x_{3}}{L \rho_{h}}+1\right) u\left(x^{\prime}\right)-\frac{x_{3}}{L \rho_{h}} w\left(x^{\prime}\right) & \text { if }\left\{\begin{array}{l}
\left(x^{\prime}, x_{3}\right) \in \Omega_{b}, \\
\left|u\left(x^{\prime}\right)-w\left(x^{\prime}\right)\right| \leq t_{k},
\end{array}\right. \\
w\left(x^{\prime}\right) & \text { if } \quad\left(x^{\prime}, x_{3}\right) \in \Omega_{s} \text { or }\left\{\begin{array}{l}
\left(x^{\prime}, x_{3}\right) \in \Omega_{b}, \\
\left|u\left(x^{\prime}\right)-w\left(x^{\prime}\right)\right|>t_{k} .
\end{array}\right.\end{cases}
$$


Clearly, $u^{k} \in S B V(\Omega), u^{k}=w$ a.e. in $\Omega_{s}$ and $\left\|u^{k}\right\|_{L^{\infty}(\Omega)} \leq M$ so that $u^{k} \in \mathcal{C}_{w}(\Omega)$ is admissible. Then for each $k \in \mathbb{N}$,

$$
\begin{gathered}
\int_{\Omega_{f}}\left(\left|\nabla^{\prime} u^{k}-\epsilon_{0}^{\prime}\right|^{2}+\frac{1}{\varepsilon_{n}^{2}}\left|\partial_{3} u^{k}\right|^{2}\right) d x=L \int_{\omega}\left|\nabla^{\prime} u-\epsilon_{0}^{\prime}\right|^{2} d x^{\prime}, \\
\int_{\Omega_{f} \cap J_{u^{k}}}\left|\left(\left(\nu_{u^{k}}\right)^{\prime}, \frac{1}{\varepsilon_{n}}\left(\nu_{u^{k}}\right)_{3}\right)\right| d \mathcal{H}^{2}=L \mathcal{H}^{1}\left(J_{u}^{\prime}\right),
\end{gathered}
$$

and

$$
\begin{aligned}
& \int_{\Omega_{b}}\left(\varepsilon_{n}^{2}\left|\nabla^{\prime} u-\epsilon_{0}^{\prime}\right|^{2}\right.\left.+\left|\partial_{3} u\right|^{2}\right) d x \\
&= \frac{1}{L \rho_{h}} \int_{\left\{|u-w| \leq t_{k}\right\}}(u-w)^{2} d x^{\prime}+L \rho_{h} \varepsilon_{n}^{2} \int_{\left\{|u-w|>t_{k}\right\}}\left|\nabla^{\prime} w-\epsilon_{0}^{\prime}\right|^{2} d x^{\prime} \\
&+\varepsilon_{n}^{2} \int_{\left\{|u-w| \leq t_{k}\right\} \times\left(-L \rho_{h}, 0\right)}\left|\left(\frac{x_{3}}{L \rho_{h}}+1\right) \nabla^{\prime} u-\frac{x_{3}}{L \rho_{h}} \nabla^{\prime} w-\epsilon_{0}^{\prime}\right|^{2} d x \\
& \underset{n \rightarrow \infty}{\longrightarrow} \frac{1}{L \rho_{h}} \int_{\left\{|u-w| \leq t_{k}\right\}}(u-w)^{2} d x^{\prime} .
\end{aligned}
$$

It remains to compute the surface energy in the bonding layer. To this end, we observe that

$$
J_{u^{k}} \cap \Omega_{b} \subset\left[J_{u}^{\prime} \times\left[-L \rho_{h}, 0\right]\right] \cup\left[\left\{|u-w|>t_{k}\right\} \times\{0\}\right] \cup\left[\partial^{*}\left\{|u-w| \leq t_{k}\right\} \times\left[-L \rho_{h}, 0\right]\right],
$$

where $\partial^{*} E$ stands for the reduced boundary of the set of finite perimeter $E$ (see (Ambrosio et al., 2000, Definition 3.54)). Then, for each $k \in \mathbb{N}$,

$$
\begin{gathered}
\int_{\Omega_{b} \cap J_{u^{k}}}\left|\left(\varepsilon_{n}\left(\nu_{u^{k}}\right)^{\prime},\left(\nu_{u^{k}}\right)_{3}\right)\right| d \mathcal{H}^{2} \\
\leq L \rho_{h} \varepsilon_{n} \mathcal{H}^{1}\left(J_{u}^{\prime}\right)+\mathcal{H}^{2}\left(\left\{|u-w|>t_{k}\right\}\right)+L \rho_{h} \varepsilon_{n} \mathcal{H}^{1}\left(\partial^{*}\left\{|u-w| \leq t_{k}\right\}\right) \\
\underset{n \rightarrow \infty}{\longrightarrow} \mathcal{H}^{2}\left(\left\{|u-w|>t_{k}\right\}\right) .
\end{gathered}
$$

Gathering (B.11), (B.12), (B.13), (B.14) yields, for each $k \in \mathbb{N}$,

$$
\begin{aligned}
\limsup _{n \rightarrow \infty} \mathcal{E}_{\varepsilon_{n}}\left(u^{k}\right) \leq L \mu_{f} \int_{\omega}\left|\nabla^{\prime} u\right|^{2} d x^{\prime}+\frac{L \mu_{b}}{h_{f} h_{b}} \int_{\left\{|u-w| \leq t_{k}\right\}}(u-w)^{2} d x^{\prime} & \\
& +L G_{f} \mathcal{H}^{1}\left(J_{u}^{\prime}\right)+\frac{L G_{b}}{h_{f}} \mathcal{H}^{2}\left(\left\{|u-w|>t_{k}\right\}\right) .
\end{aligned}
$$

Letting $k \rightarrow+\infty$ and using the monotone convergence theorem leads to

$$
\begin{aligned}
\limsup _{k \rightarrow \infty} \limsup _{n \rightarrow \infty} \mathcal{E}_{\varepsilon_{n}}\left(u^{k}\right) \leq L \mu_{f} \int_{\omega}\left|\nabla^{\prime} u\right|^{2} d x^{\prime}+\frac{L \mu_{b}}{h_{f} h_{b}} \int_{\left\{|u-w| \leq u_{d}\right\}}(u-w)^{2} d x^{\prime} & \\
& +L G_{f} \mathcal{H}^{1}\left(J_{u}^{\prime}\right)+\frac{L G_{b}}{h_{f}} \mathcal{H}^{2}\left(\left\{|u-w|>u_{d}\right\}\right) .
\end{aligned}
$$

Finally, thanks to a diagonalization procedure, it is possible to find a sequence $k_{n} \nearrow \infty$ such that

$$
\limsup _{n \rightarrow+\infty} \mathcal{E}_{\varepsilon_{n}}\left(\bar{u}_{n}\right) \leq \mathcal{E}_{0}(u)
$$

with $\bar{u}_{n}:=u^{k_{n}}$, which completes the proof of the proposition.

We end this section by pointing out that there is a connection, at the conceptual level, between the derivation of the cohesive energy in the argument we just presented and the derivation of the cohesive energy in the works by Dal Maso and Iurlano (2013), Iurlano (2012), and Focardi and Iurlano (2013). 
Ambrosio, L., Fusco, N., Pallara, D., 2000. Functions of Bounded Variation and Free Discontinuity Problems. Oxford University Press, Oxford.

Ambrosio, L., Tortorelli, V. M., Dec. 1990. Approximation of functionals depending on jumps by elliptic functional via $\Gamma$ convergence. Communications on Pure and Applied Mathematics 43 (8), 999-1036.

URL http://doi.wiley.com/10.1002/cpa.3160430805

Ambrosio, L., Tortorelli, V. M., 1992. On the approximation of Free Discontinuity Problems. Bollettino dell'Unione Matematica Italiana 7 (6-B), 105-123.

Ansini, N., 2004. The nonlinear sieve problem and applications to thin films. Asymptotic Analysis 39, $113-145$.

Ansini, N., Babadjian, J.-F., Zeppieri, C. I., May 2007. The Neumann Sieve Problem and Dimensional Reduction: a Multiscale Approach. Mathematical Models and Methods in Applied Sciences 17 (05), 681-735.

URL http://www. worldscientific.com/doi/abs/10.1142/S0218202507002078

Babadjian, J.-F., Jan. 2006. Quasistatic evolution of a brittle thin film. Calculus of Variations and Partial Differential Equations $26(1), 69-118$.

URL http://www.springerlink.com/index/10.1007/s00526-005-0369-y

Balay, S., Brown, J., Buschelman, K., Eijkhout, V., Gropp, W., Kaushik, D., Knepley, M., Mcinnes, L. C., Smith, B., Zhang, H., 2012. PETSc Users Manual. Tech. Rep. June, Argonne National Laboratory.

Bhattacharya, K., Fonseca, I., Francfort, G. A., Feb. 2002. An Asymptotic Study of the Debonding of Thin Films. Archive for Rational Mechanics and Analysis 161 (3), 205-229.

URL http://link. springer.com/10.1007/s002050100177

Bouchitte, G., Fonseca, I., Leoni, G., 2002. A Global Method for Relaxation in $W^{1, p}$ and in $S B V^{p}$. Archive for Rational Mechanics and Analysis 165, 187-242.

Bourdin, B., Francfort, G., Marigo, J.-J., 2000. Numerical experiments in revisited brittle fracture. J. Mech. Phys. Solids 48, $787-826$.

Bourdin, B., Francfort, G. A., Marigo, J.-J., 2008. The Variational Approach to Fracture. Journal of Elasticity 91 (1-3), 5-148. URL http://www.springerlink.com/index/10.1007/s10659-007-9107-3

Braides, A., 1998. Approximation of free-discontinuity problems. Springer.

URL http://books. google.com/books?hl=en\&lr=\&id=5Ks3FY29MtoC\&oi=fnd\&pg=PR13\&dq=Approximation+of+free+ discontinuity+problems\&ots=yBq71WkNYq\&sig=0sX5IVCJw2fu0ZvwnCwPhbnUEDY

Braides, A., Fonseca, I., Jan. 2001. Brittle Thin Films. Applied Mathematics and Optimization 44 (3), $299-323$.

URL http://link.springer.com/10.1007/s00245-001-0022-x

Chambolle, A., Jul. 2004. An approximation result for special functions with bounded deformation. Journal de Mathématiques Pures et Appliquées 83 (7), 929-954. URL http://linkinghub.elsevier.com/retrieve/pii/S0021782404000285

Chambolle, A., Francfort, G. A., Marigo, J.-J., Apr. 2010. Revisiting Energy Release Rates in Brittle Fracture. Journal of Nonlinear Science 20 (4), 395-424.

URL http://www.springerlink.com/index/10.1007/s00332-010-9061-2http://link.springer.com/10.1007/ s00332-010-9061-2

Chambolle, A., Giacomini, A., Ponsiglione, M., Nov. 2007. Crack Initiation in Brittle Materials. Archive for Rational Mechanics and Analysis 188 (2), 309-349.

URL http://www.springerlink.com/index/10.1007/s00205-007-0080-6

Ciarlet, P. G., 1997. Mathematical Elasticity Volume II: Theory of Plates, series stu Edition. North-Holland, Amsterdam.

Corson, F., Henry, H., Adda-Bedia, M., 2010. A model for hierarchical patterns under mechanical stresses. Philosophical Magazine 90 (1-4), 357-373.

URL http://www.tandfonline.com/doi/abs/10.1080/14786430903196665

Crosby, K. M., Bradley, R. M., 1997. Fragmentation of thin films bonded to solid substrates: Simulations and a mean-field theory. Physical Review E 55 (5), 6084-6091.

Dal Maso, G., Francfort, G. A., Toader, R., 2005. Quasistatic Crack Growth in Nonlinear Elasticity. Archive for Rational Mechanics and Analysis 176, 165-225.

Dal Maso, G., Iurlano, F., 2013. Fracture models as $\Gamma$-limits of damage models. Communications on Pure and Applied Mathematics 12 (4).

URL http://digitallibrary.sissa.it/handle/1963/4225

Fan, X., Zhang, W., Wang, T., Liu, G., Zhang, J., May 2011. Investigation on periodic cracking of elastic film/substrate system by the extended finite element method. Applied Surface Science 257 (15), 6718-6724.

URL http://linkinghub.elsevier.com/retrieve/pii/S0169433211003199

Focardi, M., Iurlano, F., 2013. Ambrosio-Tortorelli approximation of cohesive fracture models in linearized elasticity, to appear. URL http://digitallibrary.sissa.it/handle/1963/6615

Francfort, G. A., Marigo, J.-J., 1998. Revisiting Brittle Fracture as an Energy Minimization Problem. Journal of the Mechanics and Physics of Solids 46, 1319-1342.

Giacomini, A., 2005. Ambrosio-Tortorelli approximation of quasi-static evolution of brittle fractures. Calc. Var. Partial Dif. $22(2), 129-172$.

Goehring, L., Conroy, R., Akhter, A., Clegg, W. J., Routh, A. F., 2010. Evolution of mud-crack patterns during repeated drying cycles. Soft Matter 6 (15), 3562.

URL http: //xlink.rsc.org/?DOI=b922206e

Groisman, A., Kaplan, E., 1994. An Experimental Study of Cracking Induced by Desiccation . Europhysics Letters 25 (6), 415-420. 
Handge, U. A., 2002. Analysis of a shear-lag model with nonlinear elastic stress transfer for sequential cracking. Journal of Materials Science 37, 4775-4782.

Hutchinson, J. W., Suo, Z., 1992. Mixed mode cracking in layered materials. Advances in Applied Mechanics 29.

Iurlano, F., 2012. Fracture and Plastic Models as Gamma-Limits of Damage Models Under Different Regimes. Adv. Calc. Var. $6,165-189$.

URL http://cvgmt.sns.it/media/doc/paper/1684/Iur-01_06_12.pdf

León Baldelli, A. A., Bourdin, B., Marigo, J.-J., Maurini, C., May 2013. Fracture and debonding of a thin film on a stiff substrate: analytical and numerical solutions of a one-dimensional variational model. Continuum Mechanics and Thermodynamics 25 (24), 243-268.

URL http://www.springerlink.com/index/10.1007/s00161-012-0245-x

Leung, K., Néda, Z., Jul. 2000. Pattern formation and selection in quasistatic fracture. Physical review letters 85 (3), $662-5$. URL http://www.ncbi.nlm.nih.gov/pubmed/10991365

Liang, J., May 2003. Evolving crack patterns in thin films with the extended finite element method. International Journal of Solids and Structures 40 (10), 2343-2354. URL http://linkinghub.elsevier.com/retrieve/pii/S0020768303000957

Lions, J.-L., 1973. Perturbations Singulieres dans les Problemes aux Limites. Springer-Verlag, Berlin, Heidelberg, New York.

McGuigan, A. P., Briggs, G. A. D., Burlakov, V. M., Yanaka, M., Tsukahara, Y., 2003. An elastic-plastic shear lag model for fracture of layered coatings. Thin Solid Films 424, 219-223.

Mesgarnejad, A., Bourdin, B., Khonsari, M., 2013. A variational approach to the fracture of brittle thin films subject to outof-plane loading, to appear.

URL http://www.sciencedirect.com/science/article/pii/S0022509613000902

Mielke, A., 2005. Evolution of rate-independent systems. In: Dafermos, C., Feireisl, E. (Eds.), Handbook of differential equations: Evolutionary Equations. Vol. II. North-Holland, pp. 461-559.

Mumford, D., Shah, J., Jul. 1989. Optimal approximations by piecewise smooth functions and associated variational problems. Communications on Pure and Applied Mathematics 42 (5), 577-685. URL http://doi.wiley.com/10.1002/cpa.3160420503

Munson, T., Sarich, J., Wild, S., Benson, S., McInnes, L. C., 2012. TAO 2.1 Users Manual. Tech. rep., Mathematics and Computer Science Division, Argonne National Laboratory. URL http://www.mcs.anl.gov/tao

Pham, K., Amor, H., Marigo, J.-J., Maurini, C., Nov. 2011a. Gradient Damage Models and Their Use to Approximate Brittle Fracture. International Journal of Damage Mechanics 20 (4), 618-652. URL http://ijd.sagepub.com/cgi/doi/10.1177/1056789510386852

Pham, K., Marigo, J.-J., 2012. From the onset of damage up to the rupture: construction of the responses with damage localization for a general class of gradient damage models. Continuum Mech. Thermodyn. Doi : 10.1007/s00161-011-0228-3.

Pham, K., Marigo, J.-J., Maurini, C., 2011b. The issues of the uniqueness and the stability of the homogeneous response in uniaxial tests with gradient damage models. J. Mech. Phys. Solids 59 (6), 1163-1190.

Roubíček, T., Scardia, L., Zanini, C., Jul. 2009. Quasistatic delamination problem. Continuum Mechanics and Thermodynamics 21 (3), 223-235.

URL http://link.springer.com/10.1007/s00161-009-0106-4

Sadhukhan, S., Dutta, T., Tarafdar, S., Feb. 2011. Crack formation in composites through a spring model. Physica A: Statistical Mechanics and its Applications 390 (4), 731-740. URL http://linkinghub.elsevier.com/retrieve/pii/S0378437110008903

Santanach Carreras, E., Chabert, F., Dunstan, D. E., Franks, G. V., Sep. 2007. Avoiding "mud" cracks during drying of thin films from aqueous colloidal suspensions. Journal of colloid and interface science 313 (1), 160-8. URL http://www.ncbi.nlm.nih.gov/pubmed/17521665

Toga, K., Alaca, B., Aug. 2006. Junction formation during desiccation cracking. Physical Review E 74 (2), 021405. URL http://link.aps.org/doi/10.1103/PhysRevE.74.021405

Xia, Z. C., Hutchinson, J. W., 2000. Crack patterns in thin films. Journal of the Mechanics and Physics of Solids 48, $1107-1131$.

Yin, H. M., Paulino, G. H., Buttlar, W. G., Dec. 2008. An explicit elastic solution for a brittle film with periodic cracks. International Journal of Fracture 153 (1), 39-52.

URL http://www.springerlink.com/index/10.1007/s10704-008-9286-3 